\title{
Base change for ramified unitary groups: The strongly ramified case
}

\author{
By Corinne Blondel at Paris and Geo Kam-Fai Tam at Nijmegen
}

\begin{abstract}
We compute a special case of base change of certain supercuspidal representations from a ramified unitary group to a general linear group, both defined over a $p$-adic field of odd residual characteristic. In this special case, we require the given supercuspidal representation to contain a skew maximal simple stratum, and the field datum of this stratum to be of maximal degree, tamely ramified over the base field, and quadratic ramified over its subfield fixed by the Galois involution that defines the unitary group. The base change of this supercuspidal representation is described by a canonical lifting of its underlying simple character, together with the base change of the level-zero component of its inducing cuspidal type, modified by a sign attached to a quadratic Gauss sum defined by the internal structure of the simple character. To obtain this result, we study the reducibility points of a parabolic induction and the corresponding module over the affine Hecke algebra, defined by the covering type over the product of types of the given supercuspidal representation and of a candidate of its base change.
\end{abstract}

\section{Introduction}

The local Langlands correspondence for a general linear group over a non-Archimedean local field $F$ is, roughly speaking, a parametrization of its irreducible admissible representations in terms of representations of the Weil-Deligne group of $F$. An extensive study of this correspondence involves certain important invariants, e.g., L- and epsilon-factors (see [23, 24] for the characteristic 0 case, [33] for the positive characteristic case, and [45] for a recent proof). Using moreover the processes of automorphic induction and base change [5,26], one obtains an explicit description of the correspondence in the essentially tame case [14-16].

The corresponding author is Geo Kam-Fai Tam.

This project is partially supported by the European Research Council under the European Community's Seventh Framework Programme (FP7/2007-2013)/ERC Grant agreement no. 290766 (AAMOT). During the writing and the review process, the second author was supported by the Max Planck Institute for Mathematics and the Radboud Excellence Initiative fellowship.

(๕) BY ( 2021 Corinne Blondel and Geo Kam-Fai Tam, published by De Gruyter. This work is licensed under the Creative Commons Attribution 4.0 International License. 
When $F$ is of characteristic 0 , after $[4,28,38,42,44]$, we know that the local Langlands correspondence for classical groups is closely related to that for general linear groups, at least for discrete series representations: their L-packets are parametrized by multiplicity-free (conjugate-)self-dual representations of the Weil-Deligne group. Therefore, in principle, we can understand the correspondence for classical groups via general linear groups. For unitary groups, this theory is explained in the context of base change in [38] (for special orthogonal groups and symplectic groups, the theory is sometimes called local transfer [6]). More precisely, the Langlands parameter of an L-packet of a discrete series of a unitary group is the same as that of its base change, a representation of a general linear group. Hence describing Langlands parameters for L-packets of discrete series is equivalent to describing their base changes. This approach was adopted in $[1,2,7,8]$ for supercuspidal representations of small unitary groups.

In our present paper, we compute a special case of base change for ramified unitary groups, which complements the previous result in [53] for describing the local Langlands correspondence for packets of supercuspidal representations of unramified quasi-split unitary groups. In the previous case, we determined first the inertial class of the base change using the method developed in [13] (for symplectic groups), and then the exact base change by using Asai L-functions $[22,46]$. In the following paragraphs, we will summarize our new results and the methodology for ramified unitary groups, building on the method in [13] with a new calculation on modules of Hecke algebras, then we will explain why the previous method involving Asai L-functions fails.

Let $F$ be a non-Archimedean local field of residual characteristic $p$ where $p$ is odd, let $F / F_{\bullet}$ be a quadratic extension, let $V$ be a vector space over $F$, and $\tilde{G}=\operatorname{GL}_{F}(V)$. Suppose that $V$ is equipped with a Hermitian form defined with respect to $F / F_{\bullet}$, and let $G$ be the corresponding unitary group, the fixed-point subgroup of the Hermitian involution $\sigma$ of $\tilde{G}$. Take a supercuspidal representation $\pi$ of $G$, compactly induced from a cuspidal type which contains a simple character, or in other words, whose underlying self-dual semisimple stratum $\mathbf{s}$ is indeed simple (see $[19,36,49]$ for the related definitions). Suppose that $E$ is the field datum of the stratum, which is invariant under the Galois involution defined by $\sigma$, with fixed-point subfield $E_{\bullet}$. In our paper, we require that

$$
[E: F]=\operatorname{dim}_{F} V \text { and } E / E_{\bullet} \text { is quadratic ramified. }
$$

We call this the strongly ramified case. The latter condition actually forces $F / F_{\bullet}$ to be also quadratic ramified. For the final computation, we additionally assume that $E / F$ is tamely ramified.

We refer the full detail of constructing supercuspidal representations by cuspidal types to $[19,50]$ and only specify that, under the above conditions, a cuspidal type is indeed a character (a representation of degree 1). Suppose that the cuspidal type of a supercuspidal representation $\pi$ of $G$ is of the form $\rho \kappa_{0}$, where $\kappa_{0}$ is the $p$-primary beta-extension of a simple character $\theta$, in the sense of [13], and $\rho$ is a level zero character, which is a character of $\{ \pm 1\}$ in our situation. We now take $\tilde{\theta}$ the self-dual simple character for $\tilde{G}$ whose restriction to the $\sigma$-fixed-point subgroup is the square of $\theta$ (see (2.2)), and $\tilde{\kappa}_{0}$ its unique self-dual $p$-primary beta-extension (see Proposition 3.2 and the remark after). We also take $\tilde{\boldsymbol{\rho}}$ a self-dual level zero character of $E^{\times}$to form a cuspidal type $\tilde{\rho} \tilde{\kappa}_{0}$ and compactly induce it to a supercuspidal $\tilde{\pi}$ of $\tilde{G}$.

The following theorem is the main result of our present paper, giving the conditions for $\tilde{\pi}$ to be the base change of $\pi$, which is the only member in its L-packet in our case. To make 
sense of the definition of base change, we have to assume that $\operatorname{char}(F)=0$. Recall that we have assumed the "strongly ramified" condition and also that $E / F$ is tamely ramified.

Theorem 1.1. Under the above assumptions, suppose that the simple characters $\tilde{\theta}$ and $\theta$ are related as above (or see (2.2)), and the level zero characters $\tilde{\boldsymbol{\rho}}$ and $\rho$ are related by

$$
\left.\tilde{\boldsymbol{\rho}}\right|_{\boldsymbol{\mu}_{E}}=\left(\frac{\cdot}{\boldsymbol{\mu}_{E}}\right)^{f(E / F)-1} \text { and } \tilde{\boldsymbol{\rho}}\left(\varpi_{E}\right)=\rho(-1) \epsilon_{z}^{P}\left(\varpi_{E}, \mathbf{s}\right),
$$

where

- $\mu_{E}$ is the subgroup of $E^{\times}$of roots of unity with order coprime to $p$, and $\left(\dot{\dot{\mu_{E}}}\right)$ is the quadratic character of $\mu_{E}$,

- $\varpi_{E}$ is a chosen uniformizer of $E$, and $\epsilon_{z}^{P}\left(\varpi_{E}, \mathbf{s}\right)$ is a sign attached to a quadratic Gauss sum (see (4.20)), defined by $\varpi_{E}$ and the simple stratum $\mathbf{s}$ associated to $\theta$.

Then $\tilde{\pi}$ is the base change of $\pi$.

For example, when $\operatorname{dim} V=1$, then $E=F$. The $\operatorname{sign} \epsilon_{z}^{P}\left(\varpi_{E}, \mathbf{s}\right)$ is 1 , and the relation in Theorem 1.1 becomes

$$
\tilde{\boldsymbol{\rho}}(x)=\rho\left(x^{\sigma} x\right) \text { for all } x \in F^{\times},
$$

which is exactly the base change for characters of $\mathrm{U}_{1}$. When $\operatorname{dim} V>1$, we see that $\tilde{\boldsymbol{\rho}}$ differs from the base-change of $\rho$ by a tamely ramified character, or in other words the level-zero component of the inducing cuspidal type of $\tilde{\pi}$ is not the base change of that of $\pi$. The main idea here is that: when $\operatorname{dim} V>1$, we have to modify our base change formula by modifying the level zero component $\tilde{\rho}$ of the cuspidal type, by taking the internal structure of the positive level component $\tilde{\boldsymbol{\kappa}}_{0}$ into account. An analogous phenomenon was intensively studied in the essentially tame local Langlands correspondence for general linear groups [14-16]: the inducing type of a supercuspidal has to be modified in order to match with its Langlands parameter. Similar studies were conducted for quasi-split unramified unitary groups [53], and for symplectic groups [13]. In all these cases, the modifications are incarnated by certain characters (called rectifiers in the first case and amending characters in the second case) of elliptic tori, defined by the field data extracted from positive level components and taking effect only on level zero components, i.e., the modifying characters are all tamely ramified.

We now explain the methodology for the theorem. The first step, following a series of papers of the first author $[9,11,12]$, is to study the reducibility points of the parabolic induction

$$
\tilde{\pi}|\operatorname{det}|^{s} \rtimes \pi:=\operatorname{Ind}_{P}^{G_{W}}\left(\tilde{\pi}|\operatorname{det}|^{s} \otimes \pi\right), \quad s \in \mathbb{C},
$$

where $W$ is the Hermitian space $W=V^{-} \oplus V \oplus V^{+}$, with each $V^{-}$and $V^{+}$just $V$ as a vector space and $V^{-} \oplus V^{+}$hyperbolic, and so the unitary group $G_{W}$ contains a parabolic subgroup $P$ with Levi component $M=\tilde{G} \times G$. By [47], when this parabolic induction is reducible at a point $s \in \mathbb{R}$, then some twist of $\tilde{\pi}$ is self-dual. Assuming that $\tilde{\pi}$ itself is selfdual, we can use [38] to show that in our case, $\tilde{\pi}$ is the base change of $\pi$ if and only if $|s|=1$.

We apply the theory of covering types [20] to get a preliminary information about the reducibility: we construct a type $\lambda_{P}$ in $G_{W}$ covering $\tilde{\lambda} \otimes \lambda$, the product of a pair of types $\tilde{\lambda}$ and $\lambda$ for $\tilde{\pi}$ and $\pi$, respectively, providing the categorical equivalence

$$
\mathcal{R}^{[M, \tilde{\pi} \otimes \pi]}\left(G_{W}\right) \rightarrow \operatorname{Mod}-\mathscr{H}\left(G_{W}, \lambda_{P}\right)
$$


between the Bernstein component of the inertial class of $\tilde{\pi} \otimes \pi$ in $G_{W}$ and the module category of the Hecke algebra $\mathscr{H}\left(G_{W}, \lambda_{P}\right)$. When $\tilde{\lambda}$ is self-dual with $\tilde{\theta}$ and $\theta$ related by (2.2), we single out two candidates for $\tilde{\pi}$ that are self-dual representations in the inertial class of the base change of $\pi$, the two differing from each other by an unramified character of a certain finite order, such that $\tilde{\pi}|\operatorname{det}|^{S} \rtimes \pi$ is reducible at a point in $\mathbb{R}_{\geq 0}$.

The second step is to further study the structure of the Hecke algebra $\mathscr{H}\left(G_{W}, \lambda_{P}\right)$ as well as its modules. By [20,36], this Hecke algebra has two generators, denoted by $T_{y}$ and $T_{z}$ in this paper, each satisfying a quadratic relation

$$
T_{w}^{2}=b_{w} T_{w}+c_{w}, \quad w \in\{y, z\} .
$$

When $\left.\tilde{\boldsymbol{\rho}}\right|_{\boldsymbol{\mu}_{E}}$ is given as in Theorem 1.1, we can actually normalize $T_{w}$ by a positive scalar such that the quadratic relations for $T_{y}$ and $T_{z}$ take the similar form

$$
\left(T_{w}+\epsilon_{w}\right)\left(T_{w}-\epsilon_{w} q^{f(E / F)}\right)=0, \quad w \in\{y, z\}
$$

where each $\epsilon_{w}$ is a sign. We then compute the eigenvalues of these two generators, or equivalently the coefficients $b_{w}$, on the module corresponding to $\tilde{\pi}|\operatorname{det}|^{S} \rtimes \pi$. The product of $T_{y}$ and $T_{z}$ is equal to the image of an infinite order invertible element $Z$ in the Hecke algebra $\mathscr{H}(M, \tilde{\lambda} \otimes \lambda)$ for the Levi subgroup $M$, whose eigenvalue is computed in a different manner. We then compare the product of eigenvalues of $T_{y}$ and $T_{z}$ with that of $Z$ : when $\tilde{\pi}|\operatorname{det}|^{S} \rtimes \pi$ is reducible at $s=1$, the signs $\epsilon_{y}, \epsilon_{z}$, and that for $Z$ are respectively $\rho(-1), \epsilon_{z}^{P}\left(\varpi_{E}, \mathbf{s}\right)$, and $\tilde{\boldsymbol{\rho}}\left(\varpi_{E}\right)$ (modulo some irrelevant factors which cancel with each other in the comparison), giving the last condition for base change in Theorem 1.1. The detailed version of this result can be derived from Theorem 3.4 and Corollary 3.5.

It turns out that the coefficient $b_{y}$ is easy to compute, while $b_{z}$ involves some calculations similar to $[29,30]$ for large $p$ and [9] for $\mathrm{Sp}_{4}$, related to the structure of the simple stratum $\mathbf{s}$. To keep the computation manageable, we further assume that $E / F$ is tamely ramified. This is the condition assumed in [27,43] and the series [14-16] (see also the warning in [19, (2.2.6)]), as well as in [54] more generally. It also facilitates comparisons, by the second author in [51,52], between the essentially tame local Langlands correspondence for inner forms of general linear groups [14-17] with the twisted endoscopy theory [31,32].

Finally, we show that $\tilde{\pi}$ is indeed the base change of $\pi$ using a finiteness result from Mœglin for the possibilities of $\tilde{\pi}$ such that $\tilde{\pi}|\operatorname{det}|^{S} \rtimes \pi$ is reducible for some $s \in \mathbb{R}_{\geq 0}$ (see [38, 4. Proposition], [39, Theorem 3.2.1] for quasi-split groups, and [40, 8.3.5] for non-quasisplit groups). The result is obtained from Arthur's endoscopic character relations [3] and their generalizations in twisted endoscopy [41, XI.], which require that $\operatorname{char}(F)$ is 0 . It is possible that we could apply an approach analogous to [13, Theorem 2.5] to compute the reducibilities of $\tilde{\pi}|\operatorname{det}|^{S} \rtimes \pi$ for all $\tilde{\pi}$ and obtain a finiteness result similar to Mœglin's without the characteristic requirement, but in our strongly ramified case we rather take the above shortcut using Mœglin to simplify the discussion. (See [21] for a result on the local Langlands correspondence in positive characteristic for split classical groups without using reducibilities of induced representations.)

We now briefly explain why the previous method in [53] fails in the strongly ramified case. According to [38], there is a notion of parity of a (conjugate-)self-dual supercuspidal representation of $\mathrm{GL}_{n}$, either conjugate-orthogonal (+) or conjugate-symplectic (-) but not both. In the non-strongly ramified case, the two self-dual candidates have opposite parities because 
they differ by an unramified character $\tilde{\chi}$ such that $\tilde{\chi} \circ N_{E / F}$ is conjugate-symplectic. In this case we can determine the correct base change between the two by computing their parities using Asai L-functions for example [25]. However, in the strongly ramified case, $\tilde{\chi} \circ N_{E / F}$ is then conjugate-orthogonal, and so the two self-dual candidates have the same parity (see Section 3.4 for a detailed discussion). This explains why the previous method no longer works, and we have to rely on the complete structure of the modules over the Hecke algebra.

1.1. Notations. Let $F_{\bullet}$ be a non-Archimedean local field, with ring of integers ${ }^{\mathfrak{D}} F_{\bullet}$, its maximal ideal $\mathfrak{p}_{F_{\bullet}}$, and residue field $\mathbf{k}_{F_{\bullet}}=\mathfrak{v}_{F_{\bullet}} / \mathfrak{p}_{F_{\bullet}}$ of cardinality $q_{\bullet}$ and odd characteristic $p$. Let $F / F_{\bullet}$ be a quadratic extension, whose residue field $\mathbf{k}_{F}=\mathfrak{o}_{F} / \mathfrak{p}_{F}$ has $q$ elements, such that $q=q \bullet \bullet^{2}$ in the unramified case, and $q=q \bullet$ in the ramified case. Let $\mu_{F}$ be the subgroup of roots of unity of $F$ whose orders are coprime to $p$.

We denote $U_{F}:=\mathfrak{o}_{F}^{\times}$and $U_{F}^{k}:=1+\mathfrak{p}_{F}^{\times}$for $k \in \mathbb{Z}_{\geq 1}$. If $N_{F / F_{\bullet}}: F \rightarrow F_{\bullet}$ is the norm map, we denote $U_{F / F_{\bullet}}:=\mathfrak{v}_{F}^{\times} \cap \operatorname{ker} N_{F / F_{\bullet}}$ and $U_{F / F_{\bullet}}^{k}:=U_{F}^{k} \cap \operatorname{ker} N_{F / F_{\bullet}}$ for $k \in \mathbb{Z}_{\geq 1}$. The Galois group $\operatorname{Gal}\left(F / F_{\bullet}\right)$ is generated by an involutive automorphism $c$.

If $\Lambda: \mathbb{Z} \rightarrow S$ is a sequence into a set $S$, then we extend $\Lambda$ from $\mathbb{Z}$ to $\mathbb{R}$ by putting $\Lambda(r)=\Lambda(\lceil r\rceil)$ and $\Lambda\left(r_{+}\right)=\Lambda\left(\left\lceil r_{+}\right\rceil\right)$, where $\lceil r\rceil$ and $\left\lceil r_{+}\right\rceil$are the smallest integers $\geq r$ and $>r$, respectively.

\section{Review of known results}

2.1. Unitary groups. Let $V$ be an $F$-vector space. We denote $\tilde{A}=\tilde{A}_{V}=\operatorname{End}_{F}(V)$ and $\tilde{G}=\tilde{G}_{V}=\operatorname{Aut}_{F}(V)$. Suppose that $V$ is equipped with a non-degenerate $\left(F / F_{\bullet}, \epsilon\right)$-Hermitian form $h=h_{V}$, where $\epsilon=\epsilon_{V}= \pm 1$. If $X \mapsto \bar{X}$ is the conjugate-adjoint on $\tilde{A}$ defined by $h$, we define the adjoint anti-involution ${ }^{\alpha} X=-\bar{X}$. Note that

$$
{ }^{\alpha}(X Y)=-{ }^{\alpha} Y^{\alpha} X, \quad X, Y \in \tilde{A} .
$$

We also have the corresponding involution $\sigma: X \mapsto \bar{X}^{-1}$ on $\tilde{G}$. The subgroup $G=G_{V}=\tilde{G}^{\sigma}$ is a (connected) unitary group that we consider throughout the paper and whose Lie algebra is $A=\tilde{A}^{\alpha}$.

Given an $\mathfrak{D}_{F}$-lattice $L$ in $V$, we denote by $L^{*}$ its dual $\left\{v \in V: h(v, L) \subset \mathfrak{p}_{F}\right\}$. We call an $\mathfrak{D}_{F}$-lattice sequence $\Lambda$ in $V$ self-dual if there exists $d \in \mathbb{Z}$ such that $\Lambda(k)^{*}=\Lambda(d-k)$ for all $k \in \mathbb{Z}$. As in [50], we always normalize a self-dual lattice sequence such that

$$
d=1 \text { and its } \mathfrak{o}_{F} \text {-period } e\left(\Lambda / \mathfrak{o}_{F}\right) \text { is even. }
$$
in $\tilde{A}$ by

Given an $\mathfrak{D}_{F}$-lattice sequence $\Lambda$ in $V$, we define an $\mathfrak{o}_{F}$-lattice sequence $\tilde{\mathfrak{P}}_{\Lambda}^{k}$, for $k \in \mathbb{Z}$,

$$
\tilde{\mathfrak{R}}_{\Lambda}^{k}=\{x \in \tilde{A}: x \Lambda(m) \subseteq \Lambda(m+k) \text { for all } m \in \mathbb{Z}\} .
$$

Hence $\tilde{\mathfrak{A}}_{\Lambda}:=\tilde{\mathfrak{P}}_{\Lambda}^{0}$ is a hereditary order in $\tilde{A}$, with Jacobson radical $\tilde{\mathfrak{P}}_{\Lambda}:=\tilde{\mathfrak{P}}_{\Lambda}^{1}$. We denote by $v_{\Lambda}$ the valuation on $\tilde{A}$ associated to $\Lambda$. If $\Lambda$ is self-dual, then each $\tilde{\mathfrak{P}}_{\Lambda}^{k}$ is $\alpha$-invariant, in which case we put $\mathfrak{P}_{\Lambda}=\tilde{\mathfrak{P}}_{\Lambda}^{\alpha}$.

We also define $\tilde{U}_{\Lambda}=\tilde{U}_{\Lambda}^{0}=\tilde{\mathfrak{A}}_{\Lambda}^{\times}$and $\tilde{U}_{\Lambda}^{k}=1+\tilde{\mathfrak{P}}_{\Lambda}^{k}$ for $k \in \mathbb{Z}_{\geq 1}$. If $\Lambda$ is self-dual, then each $\tilde{U}_{\Lambda}^{k}$ is $\sigma$-invariant, in which case we put $U_{\Lambda}=\tilde{U}_{\Lambda}^{\sigma}$ and $U_{\Lambda}^{k}=\left(\tilde{U}_{\Lambda}^{k}\right)^{\sigma}$. The quotient $\mathbf{G}_{\Lambda}=U_{\Lambda} / U_{\Lambda}^{1}$ is the group of $\mathbf{k}_{F_{\bullet}}$-points of a reductive group $\mathbf{G}_{\Lambda}$ defined over $\mathbf{k}_{F_{\bullet}}$, 
which is disconnected in general. We denote by $U_{\Lambda}^{0}$ the inverse image of $\mathrm{G}_{\Lambda}^{0}$ in $U_{\Lambda}$, where $\mathrm{G}_{\Lambda}^{0}$ is the subgroup of $\mathbf{k}_{F_{\bullet}}$-points of the identity component $\mathbf{G}_{\Lambda}^{0}$ of $\mathbf{G}_{\Lambda}$.

Suppose that $\Lambda$ is a self-dual lattice sequence of the form

$$
\Lambda(0) \supseteq \Lambda(1)=\Lambda(0)^{*} \supseteq \Lambda(2)=\mathfrak{p}_{F} \Lambda(0) .
$$

Then $U_{\Lambda}$ is a maximal compact subgroup, and $U_{\Lambda}^{0}$ is the underlying maximal parahoric subgroup. When $F / F_{\bullet}$ is unramified, $\mathrm{G}_{\Lambda}^{0}$ is a product of at most two unitary groups relative to $\mathbf{k}_{F} / \mathbf{k}_{F_{\bullet}}$; while when $F / F_{\bullet}$ is ramified it is a product of at most one symplectic group and at most one special orthogonal group, both defined over $\mathbf{k}_{F}=\mathbf{k}_{F_{\bullet}}$ (see [50, Section 3.3]).

2.2. Cuspidal types. We recall from $[19,50]$ the constructions of cuspidal types for general linear groups and unitary groups. The compact inductions of these types are irreducible supercuspidal representations.

Let $\mathbf{s}=[\Lambda, r, 0, \beta]$ be either a simple stratum or the null stratum $[\Lambda, 0,0,0]$ where, in the former case, we denote $E=F[\beta]$, which is a field contained in $\tilde{A}$, such that $\Lambda$ is an $\mathfrak{o}_{E}$-lattice chain, while in the latter case, we put $E=F$ and $\Lambda(k)=\operatorname{Mat}_{n}\left(\mathfrak{p}_{F}^{k}\right)$. We denote by $\tilde{A}_{E}$ and $\tilde{G}_{E}$, respectively, the centralizer of $\beta$ in $\tilde{A}$ and $\tilde{G}$, and for $k \in \mathbb{Z}$, denote $\tilde{\mathfrak{P}}_{\Lambda, E}^{k}=\tilde{\mathfrak{P}}_{\Lambda}^{k} \cap \tilde{A}_{E}$ and $\tilde{U}_{\Lambda, E}^{k}=\tilde{U}_{\Lambda}^{k} \cap \tilde{G}_{E}$. As in [19, Chapter 3], associated to s we construct

- subrings $\tilde{\tilde{\mathfrak{H}}}=\tilde{\mathfrak{H}}_{\Lambda, \beta} \subseteq \tilde{\tilde{\mathcal{J}}}=\tilde{\widetilde{\mathcal{J}}}_{\Lambda, \beta}$ of $\tilde{A}$ and the two-sided fractional ideals $\tilde{\mathfrak{F}}^{k}=\tilde{\mathfrak{H}} \cap \tilde{\mathfrak{F}}_{\Lambda}^{k}$ and $\tilde{\widetilde{\jmath}}^{k}=\tilde{\tilde{\jmath}} \cap \tilde{\mathfrak{P}}_{\Lambda}^{k}$, for all $k \in \mathbb{Z}$,

- subgroups $\tilde{H}^{k}=\tilde{H}_{\Lambda, \beta}^{k}=\tilde{\mathfrak{S}} \cap \tilde{U}_{\Lambda}^{k} \subset \tilde{J}^{k}=\tilde{J}_{\Lambda, \beta}^{k}=\tilde{\tilde{J}} \cap \tilde{U}_{\Lambda}^{k}$ of $\tilde{G}$, for all $k \in \mathbb{Z}_{>0}$,

- $\tilde{e}(\mathbf{s}):=\tilde{e}(\Lambda, 0, \beta)$ the set of simple characters of $\tilde{H}^{1}$,

- associated to each simple character $\tilde{\theta} \in \tilde{\mathscr{C}}(\mathbf{s})$ the Heisenberg representation $\tilde{\eta}=\tilde{\eta}_{\tilde{\theta}}$ of $\tilde{J}^{1}$, an irreducible representation that restricts to a multiple of $\tilde{\theta}$ on $\tilde{H}^{1}$,

- a beta-extension $\tilde{\kappa}$ of $\tilde{\eta}$ to the subgroup $\tilde{J}=\tilde{J}_{\Lambda, \beta}=\tilde{U}_{\Lambda, E} \tilde{J}^{1}$ (note that among these extensions there is a unique one $\tilde{\kappa}_{0}$ whose determinant has order a power of $p$, called the $p$-primary beta-extension).

To construct a supercuspidal representation of $\tilde{G}$, we require that $\Lambda$ is principal, which is assumed from now on, and also $e\left(\Lambda / \mathfrak{o}_{E}\right)=2$ (note the convention in (2.1)). We take an irreducible representation $\tilde{\rho}$ of $\tilde{J}$ inflated from a cuspidal representation of

$$
\tilde{J} / \tilde{J}^{1} \cong \mathrm{GL}_{n /[E: F]}\left(\mathbf{k}_{E}\right) \text {. }
$$

The maximal simple type $\tilde{\lambda}=\tilde{\kappa} \otimes \tilde{\rho}$ extends to an irreducible representation $\tilde{\lambda}$ of $\tilde{\mathbf{J}}=E_{\tilde{G}}^{\times} \tilde{J}$, which is then compactly induced to an irreducible supercuspidal representation $\tilde{\pi}=\operatorname{cInd}_{\tilde{\mathbf{J}}} \tilde{\boldsymbol{\lambda}}$.

Note that if $\mathbf{s}$ is null, then by convention we assume that $e\left(\Lambda / \mathfrak{o}_{F}\right)=2$, and $\tilde{e}$ (s) contains only the trivial character of $\tilde{H}^{1}=\tilde{J}^{1}=\tilde{U}_{\Lambda}^{1}$. We take $\tilde{\kappa}$ of $\tilde{U}_{\Lambda}$ to be trivial, and choose $\tilde{\rho}$ to be inflated from a cuspidal representation of $\tilde{U}_{\Lambda} / \tilde{U}_{\Lambda}^{1} \cong \mathrm{GL}_{n}\left(\mathbf{k}_{F}\right)$. The extension $\tilde{\lambda}$ on $\tilde{\mathbf{J}}=F^{\times} \tilde{J}$ of $\tilde{\lambda}=\tilde{\rho}$ can be chosen by fixing a central character, and so $\tilde{\pi}=\operatorname{cInd}_{\tilde{\mathbf{J}}} \tilde{\boldsymbol{\lambda}}$ is a level zero supercuspidal representation.

Every supercuspidal representation of $\tilde{G}$ is obtained by the way above. Moreover, the maximal simple type $\tilde{\lambda}$ is determined by $\tilde{\pi}$ up to conjugacy, and so is the extended type $\tilde{\lambda}$ containing $\tilde{\lambda}$.

If $V$ is a Hermitian space, as in Section 2.1, we call a simple stratum skew if $\Lambda$ is self-dual and $\beta \in A$. In this case, all subgroups $\tilde{H}^{1}, \tilde{J}^{1}, \tilde{J}$, and $\tilde{\mathbf{J}}$ are $\sigma$-invariant. If we choose $\tilde{\theta}, \tilde{\kappa}, \tilde{\rho}$, and the extension of $\tilde{\lambda}$ to be self-dual, i.e., $\sigma$-invariant, then so is $\tilde{\pi}$. Note that the 
$p$-primary beta-extension $\tilde{\kappa}_{0}$ of $\tilde{\theta}$ is self-dual because of its uniqueness. If $\beta \neq 0$, then $-\alpha$ restricts to a Galois involution on $E$. We denote the fixed field by $E_{\bullet}$. Since $\tilde{A}_{E}$ and $\tilde{\mathfrak{P}}_{\Lambda, E}^{k}$, resp. $\tilde{G}_{E}, \tilde{U}_{\Lambda, E}$, and $\tilde{U}_{\Lambda, E}^{k}$, are invariant under $\alpha$, resp. $\sigma$, we define $A_{E / E_{\bullet}}=\tilde{A}_{E}^{\alpha}, G_{E / E_{\bullet}}=\tilde{G}_{E}^{\sigma}$, and $\mathfrak{P}_{\Lambda, E / E_{\bullet}}^{k}, U_{\Lambda, E / E_{\bullet}}, U_{\Lambda, E / E_{\bullet}}^{k}$ similarly.

When $\mathbf{S}$ is skew and semisimple, following [50], we construct

- subgroups $H^{1}:=\left(\tilde{H}^{1}\right)^{\sigma} \subseteq J^{1}:=\left(\tilde{J}^{1}\right)^{\sigma} \subset J:=\tilde{J}^{\sigma}$,

- a set $\mathscr{C}(\mathbf{s}):=\ell(\Lambda, 0, \beta)$ of semisimple characters of $H^{1}$, defined as follows: denote the subset of $\tilde{\mathscr{C}}(\mathbf{s})$ of self-dual semisimple characters by $\tilde{\mathscr{C}}(\mathbf{s})^{\sigma}$, and define $\mathcal{C}(\mathbf{s})$ to be the image set of the following map (well-defined since the group $H^{1}$ is a pro- $p$ subgroup where $p$ is odd):

$$
\tilde{e}(\mathbf{s})^{\sigma} \rightarrow \ell(\mathbf{s}), \quad \tilde{\theta} \mapsto\left(\left.\tilde{\theta}\right|_{H^{1}}\right)^{1 / 2},
$$

which turns out to be bijective since the restriction operation satisfies the properties of Glauberman correspondence (see [50, Section 5.3] for details),

- associated to each semisimple character $\theta \in \mathscr{C}(\mathbf{s})$ the Heisenberg representation $\eta=\eta_{\theta}$ of $J^{1}$, an irreducible representation that restricts to a multiple of $\theta$ on $H^{1}$,

- a beta-extension $\kappa$ of $\eta$ to the subgroup $J=U_{\Lambda, E / E_{\bullet}} J^{1}$ (note that among these extensions there is a unique one $\kappa_{0}$ whose determinant has order a power of $p$, called the p-primary beta-extension).

To construct a supercuspidal representation of $G$, from now on we suppose that $G_{E / E}$. has a compact center and the parahoric subgroup $U_{\Lambda, E / E_{\bullet}}^{0}$ is maximal in $G_{E / E_{\bullet}}$. We take an irreducible representation $\rho$ of $J$ inflated from a cuspidal representation $\bar{\rho}$ of $G=J / J^{1}$, a (possibly non-connected) classical group over the finite field $\mathbf{k}_{F_{\bullet}}$. (A cuspidal representation of a disconnected $G$ means that its restriction to $G^{0}$ is a sum of conjugates of a cuspidal representation.) The product $\lambda=\kappa \otimes \rho$ is then a cuspidal type, and is compactly induced to an irreducible supercuspidal representation $\pi=\operatorname{cInd}_{J}^{G} \lambda$. If $\mathbf{s}$ is null, then by definition $\mathcal{C}(\mathbf{s})$ contains only the trivial character of $U_{\Lambda}^{1}$. We again take $\kappa$ to be trivial, and so $\pi=\operatorname{cInd}_{J}^{G} \rho$ is a level zero supercuspidal representation.

Remark 2.1. Note that $G_{E / E_{\bullet}}$ has a compact center except precisely when it has a factor isomorphic to the split $\mathrm{SO}_{2}$, (which is just a $\mathrm{GL}_{1}$ ), which does not happen in our case. Also, for the parahoric subgroup $U_{\Lambda, E / E_{\bullet}}^{0}$ to be maximal, it is not enough to just assume that the order $\mathfrak{P}_{\Lambda, E}^{0}$ is maximal, since there exists such a maximal order whose corresponding parahoric subgroup is not maximal. See [36, Appendix] for details.

2.3. Covering types. To proceed, we require a larger unitary group $G_{W}$ defined on the space $W=V \perp Z$. Here $Z=Z_{-} \oplus Z_{+}$is an $\epsilon$-Hermitian space, with $Z_{+}$being a finitedimensional vector space over $F$, and $Z_{-}$is the dual space of $Z_{+}$with respect to the form

$$
h_{Z}\left((z-, z+),\left(w_{-}, w_{+}\right)\right)=\left(z_{-}, w_{+}\right)+\epsilon \cdot{ }^{c}\left(w_{-}, z_{+}\right) \text {, }
$$

for $z_{-}, w_{-} \in Z_{-}$and $z_{+}, w_{+} \in Z_{+}$. The $\epsilon$-Hermitian form on $W$ is $h_{W}=h \perp h_{Z}$. We denote the Lie algebra of $G_{W}$ by $A_{W}$, the subspace of fixed points of $\tilde{A}_{W}=\operatorname{End}_{F}(W)$ by the adjoint anti-involution defined by $h_{W}$. 
We denote by $M$ the Levi subgroup of block diagonal matrices in $G_{W}$, isomorphic to $\tilde{G}_{Z_{-}} \times G_{V}$. We denote by $P$ the subgroup of block upper triangular matrices leaving invariant the flag $Z_{-} \subset V \subset W$, with unipotent radical $U$ and the opposite $U^{-}$. We denote by $i_{M}: M \rightarrow G_{W}$ the embedding $(g, h) \mapsto \operatorname{diag}\left(g, h,{ }^{\sigma} g\right)$ for $g \in \tilde{G}, h \in G$, and abbreviate

$$
(X, Y)^{+}=\left[\begin{array}{ccc}
I & X & Y \\
& I & { }^{\alpha} X \\
& & I
\end{array}\right] \in U, \quad(X, Y)^{-}=\left[\begin{array}{ccc}
I & & \\
{ }^{\alpha} X & I & \\
Y & X & I
\end{array}\right] \in U^{-} .
$$

We can check that these matrices satisfy the relation

$$
X^{\alpha} X=Y-{ }^{\alpha} Y \text {. }
$$

If $\Lambda_{+}$is an $\mathfrak{v}_{F}$-lattice chain in $Z_{+}$, we define

$$
\Lambda_{-}(k)=\left\{z_{-} \in Z_{-}:\left(z_{-}, \Lambda_{+}(1-k)\right) \subset \mathfrak{p}_{F}\right\}, \quad k \in \mathbb{Z} .
$$

If $\beta_{+} \in \tilde{A}_{Z_{+}}$, we define $\beta_{-} \in \tilde{A}_{Z_{-}}$by

$$
\left(\beta_{-} z_{-}, z_{+}\right)=-^{c}\left(z_{-}, \beta_{+} z_{+}\right), \quad z_{-} \in Z_{-}, z_{+} \in Z_{+} .
$$

In this general setting, let $\lambda_{V}$ be a cuspidal type in $G_{V}$ and let $\tilde{\lambda}_{Z_{-}}$be a cuspidal type in $\tilde{G}_{Z_{-}}$as in Section 2.2. A covering type in $G_{W}$ for the cuspidal type $\tilde{\lambda}_{Z_{-}} \otimes \lambda_{V}$ in $M$ is constructed in [36], providing a type for the Bernstein component of the smooth dual of $G_{W}$ that contains a representation whose cuspidal support comes from a supercuspidal representation of $M$ containing $\tilde{\lambda}_{Z_{-}} \otimes \lambda_{V}$. The use of covering types in the study of parabolic induction is briefly recalled in the next subsection. In the context of base change or of the description of L-packets, most covering types are irrelevant; the relevant ones are those in which the cuspidal type $\tilde{\lambda}_{Z_{-}}$bears a strong relation with $\lambda_{V}$. In the present work we focus on the case when the stratum $\mathbf{s}$ underlying $\lambda_{V}$ is skew simple. In this case, and when studying base change, the relevant covering types are those for which the space $Z_{-}$has the same dimension as $V$ and the cuspidal type $\tilde{\lambda}_{Z_{-}}$is built from a simple character that transfers in $V$ to the square of the simple character underlying $\lambda_{V}$ (or see (2.4) for the precise relation).

So we proceed to construct covering types for $G_{W}$ precisely in that case.

Assumptions 2.2. Let us assume the following from now on.

- $Z_{-}$is identified to $V$ as a vector space, and we identify $Z_{+}$with $Z_{-}$by $h=h_{V}$.

- $\beta_{-}=\beta=\beta_{+}$; in particular, it means that $\beta$ is self-dual: ${ }^{\alpha} \beta=\beta$.

- $\Lambda_{-}=\Lambda$, which is an ${ }^{\mathfrak{D}_{E}}$-lattice chain, with $E=F[\beta]$. We moreover assume that $\Lambda_{V}$ is principal and its $\mathfrak{D}_{E}$-period is 2 (hence its $\mathfrak{D}_{F}$-period is $2 e(E / F)$ ).

We define a self-dual $\mathfrak{D}_{E}$-lattice sequence $\mathfrak{m}$ in $W=V \perp Z$ of $\mathfrak{o}_{E}$-period 6 (hence of $\mathfrak{D}_{F}$-period $\left.6 e(E / F)\right)$ by

$$
\mathfrak{m}(k)=\Lambda_{-}\left(\frac{k-1}{3}\right) \oplus \Lambda\left(\frac{k}{3}\right) \oplus \Lambda_{+}\left(\frac{k+1}{3}\right), \quad k \in \mathbb{Z},
$$

and two maximal self-dual $\mathfrak{D}_{E}$-lattice sequences $\mathfrak{M}^{w}$ in $W$, where $w \in\{y, z\}$ and both of ${ }^{\mathfrak{D}}{ }_{E}$-period 2, such that the set of lattices in the sequence $\mathfrak{M}^{y}$ is

$$
\left\{\Lambda_{-}(k) \oplus \Lambda(k) \oplus \Lambda_{+}(k): k \in \mathbb{Z}\right\},
$$


while those in $\mathfrak{M}^{z}$ is

$$
\left\{\Lambda_{-}(k) \oplus \Lambda(k) \oplus \Lambda_{+}(k+1), \Lambda_{-}(k) \oplus \Lambda(k+1) \oplus \Lambda_{+}(k+1): k \in \mathbb{Z}\right\} .
$$

Given a skew simple or null stratum $\mathbf{s}=[\Lambda, r, 0, \beta]$, we hence have corresponding skew semisimple strata $\mathbf{s}_{\mathfrak{m}}=\left[\mathfrak{m}, r_{\mathfrak{m}}, 0, \beta\right]$ and $\mathbf{s}_{w}=\left[\mathfrak{M}^{w}, r_{w}, 0, \beta\right]$, with $w \in\{y, z\}$, where $\beta$ is embedded into $\tilde{A}_{W}$ as the block-diagonal matrix with diagonal blocks $(\beta, \beta, \beta)$. We now follow $[19,50]$ to define, for $\mathscr{L}=\mathfrak{m}$ or $\mathfrak{M}^{w}$, with $w \in\{y, z\}$,

- subgroups $H_{\mathfrak{L}}^{1} \subseteq J_{\mathfrak{L}}^{1} \subset J_{\mathfrak{R}}$ in $G_{W}$,

- semisimple characters $\theta_{\mathfrak{R}}$ on $H_{\mathfrak{L}}^{1}$, such that they satisfy a transfer relation with each other, as in [19, (3.6)], [49, Section 3.5],

- beta-extensions $\kappa_{\mathfrak{M}} w$ of $\theta_{\mathfrak{M}} w$ on $J_{\mathfrak{M}} w$, and $\kappa_{\mathfrak{m}}^{w}$ of $\theta_{\mathfrak{m}}$ on $J_{\mathfrak{m}}$, such that $\kappa_{\mathfrak{M}} w$ and $\kappa_{\mathfrak{m}}^{w}$ are compatible in the sense of [50, Lemma 4.3],

- unique $p$-primary beta extension $\left(J_{\mathfrak{M}} w, \kappa_{\mathfrak{M}} w, 0\right)$, and the one $\left(J_{\mathfrak{m}}, \kappa_{\mathfrak{m}, 0}^{w}\right)$ compatible with $\kappa_{\mathfrak{M}}^{w}, 0$ (note that $\kappa_{\mathfrak{m}, 0}^{w}$ is in general not the $p$-primary beta extension $\kappa_{\mathfrak{m}, 0}$ of $\theta_{\mathfrak{m}}$ ).

Since the decomposition $W=V \perp\left(Z_{-} \oplus Z_{+}\right)$is properly subordinate to the stratum $\mathbf{s}_{\mathfrak{m}}$, the subgroups $H_{\mathfrak{m}}^{1}, J_{\mathfrak{m}}^{1}$, and $J_{\mathfrak{m}}$ admit an Iwahori decomposition of the form

$$
K=K^{-} K_{M} K^{+}, \quad \text { where } K_{M}=K \cap M, K^{+}=K \cap U, \text { and } K^{-}=K \cap U^{-},
$$

for $K$ being one of the compact subgroups just mentioned, and the product can be taken in any order. We can then show from [50, Corollary 5.11 and Proposition 5.5] that $H_{M}^{1}:=\tilde{H}^{1} \times H^{1}$ and

$$
\left.\theta_{\mathfrak{m}}\right|_{H_{M}^{1}}=\tilde{\theta}_{\Lambda_{-}} \otimes \theta_{\Lambda},
$$

where $\tilde{\theta}_{\Lambda_{-}}$and $\theta_{\Lambda}$ are simple characters related under the correspondence (2.2), i.e.,

$$
\theta_{\Lambda}=\left(\left.\tilde{\theta}_{\Lambda_{-}}\right|_{H^{1}}\right)^{1 / 2}
$$

To construct a covering type, we define

- the following subgroups:

$$
\begin{aligned}
H_{P}^{1} & =\tilde{H}_{\mathfrak{m}}^{1}\left(\tilde{J}_{\mathfrak{m}}^{1} \cap U\right) \cap G_{W}=H_{\mathfrak{m}}^{1}\left(J_{\mathfrak{m}}^{1} \cap U\right), \\
J_{P}^{1} & =\tilde{H}_{\mathfrak{m}}^{1}\left(\tilde{J}_{\mathfrak{m}}^{1} \cap P\right) \cap G_{W}=H_{\mathfrak{m}}^{1}\left(J_{\mathfrak{m}}^{1} \cap P\right), \\
J_{P} & =\tilde{H}_{\mathfrak{m}}^{1}\left(\tilde{J}_{\mathfrak{m}} \cap P\right) \cap G_{W}=H_{\mathfrak{m}}^{1}\left(J_{\mathfrak{m}} \cap P\right),
\end{aligned}
$$

and also denote $J_{P}^{ \pm}=J_{P} \cap U^{ \pm}$,

- a simple character $\theta_{P}$ on $H_{P}^{1}$ by extending $\theta_{\mathfrak{m}}$ trivially to $J_{\mathfrak{m}}^{1} \cap U$, and the unique representation $\eta_{P}$ on $J_{P}^{1}$ containing $\theta_{P}$ (by [50, Lemma 5.12]),

- for $w \in\{y, z\}$, an extension $\kappa_{P}^{w}$ of $\eta_{P}$ to $J_{P}^{1}$ that is the restriction of $\kappa_{\mathfrak{m}}^{w}$ to the subspace of $\left(J_{\mathfrak{m}}^{1} \cap U\right)$-fixed vectors of $\eta_{\mathfrak{m}}$ (which can be also characterized by the relation $\left.\kappa_{\mathfrak{m}}^{w} \cong \operatorname{Ind}_{J_{P}}^{J_{\mathfrak{m}}} \kappa_{P}^{w}\right)$.

With our construction of $\mathfrak{m}$, the decomposition $W=V \perp\left(Z_{-} \oplus Z_{+}\right)$is moreover exactly subordinate to $\mathbf{s}_{\mathfrak{m}}$. By [50, Proposition 6.3], if we write $\kappa_{P}^{w} \mid J_{M}=\tilde{\kappa}^{w} \otimes \kappa^{w}$, then $\tilde{\kappa}^{w}$ and $\kappa^{w}$ are beta-extensions of $\tilde{\theta}$ and $\theta$, respectively, and $\tilde{\kappa}^{w}$ is self-dual (with respect to $\sigma$ ), see [50, Corollary 6.10]. 
We then choose a (product of) cuspidal representation $\rho_{M}=\tilde{\rho} \otimes \rho$ of $J_{P}$, inflated from $J_{P} / J_{P}^{1} \cong J_{M} / J_{M}^{1}$. (At this moment, there is no relation between $\tilde{\rho}$ and $\rho$.) Define

$$
\lambda_{P}^{w}=\kappa_{P}^{w} \otimes \rho_{M}
$$

which is a covering type over $\lambda_{M}^{w}:=\left.\lambda_{P}^{w}\right|_{J_{M}}$ by [36]. Finally, we denote by

$$
\pi_{M}^{w}=\tilde{\pi}^{w} \otimes \pi^{w}
$$

a supercuspidal representation of $M=\tilde{G}_{Z} \times G_{V}$ containing the maximal type

$$
\lambda_{M}^{w}=\tilde{\lambda}^{w} \otimes \lambda^{w},
$$

where $\tilde{\lambda}^{w}=\tilde{\kappa}^{w} \otimes \tilde{\rho}$ and $\lambda^{w}=\kappa^{w} \otimes \rho$.

2.4. Covers and Hecke algebras. At the beginning of this subsection, we use $G$ to denote the $F$-points of a connected reductive group over $F$, and will later switch back to denote a unitary group as in previous subsections. We recall the following notions from [20].

- Suppose that $G$ contains a parabolic subgroup $P$ with Levi component $M$. If $\pi_{M}$ is a supercuspidal representation of $M$, we denote by $\mathfrak{s}=\left[M, \pi_{M}\right]_{G}$ the inertial class of $\pi_{M}$, and by $\mathcal{R}^{\mathfrak{s}}(G)$ the full subcategory of representations of $G$ whose irreducible subquotients are those of the normalized parabolic induction $\iota_{P}^{G} \tau:=\operatorname{Ind}_{P}^{G}\left(\tau \Delta_{P}^{1 / 2}\right)$, where $\tau$ ranges over representations in $\mathfrak{s}$ and $\Delta_{P}$ is the modulus character of $P$.

- Suppose that $K$ is a compact open subgroup of $G$. We fix a Haar measure on $G$ such that $K$ has volume 1 . Given a representation $\lambda$ of $K$ on a finite-dimensional $\mathbb{C}$-vector space $W$, we denote by $\mathscr{H}(G, \lambda)$ the associated Hecke algebra, which is the space of compactly supported functions $f: G \rightarrow \operatorname{End}_{\mathbb{C}}(W)$ satisfying

$$
f\left(k_{1} g k_{2}\right)=\lambda\left(k_{1}\right) \circ f(g) \circ \lambda\left(k_{2}\right) \quad \text { for all } k_{1}, k_{2} \in K \text { and } g \in G,
$$

with an associative $\mathbb{C}$-algebra structure under the convolution

$$
f_{1} * f_{2}(g)=\int_{G} f_{1}(x) f_{2}\left(x^{-1} g\right) d x \quad \text { for all } g \in G .
$$

The support of every element in $\mathscr{H}(G, \lambda)$ lies in the intertwining set

$$
I_{G}(\lambda):=\left\{g \in G: \operatorname{Hom}_{K \cap g K g^{-1}}\left(\lambda,{ }^{g} \lambda\right) \neq 0\right\} .
$$

We call $(K, \lambda)$ an s-type if, for an irreducible representation $\tau$ of $G$,

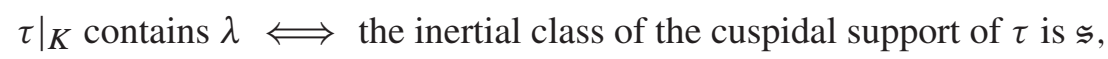

in which case we have an equivalence of categories

$$
\mathcal{M}: \mathcal{R}^{\mathfrak{s}}(G) \rightarrow \operatorname{Mod}-\mathscr{H}(G, \lambda), \quad \tau \mapsto \operatorname{Hom}_{K}(\lambda, \tau) .
$$

We now return to our notations in the previous subsection, suppose that $\pi_{M}$ contains a type $\left(J_{M}, \lambda_{M}\right)$, and $\left(J_{P}, \lambda_{P}\right)$ is a $G_{W}$-cover of $\left(J_{M}, \lambda_{M}\right)$ (e.g., $\lambda_{P}=\lambda_{P}^{w}$, for $\left.w \in\{y, z\}\right)$. In particular, we have

$$
\left.\lambda_{P}\right|_{J_{M}}=\lambda_{M}
$$


Since $\left(J_{M}, \lambda_{M}\right)$ is an $\left[M, \pi_{M}\right]$-type, we obtain:

- that $\left(J_{P}, \lambda_{P}\right)$ is a $\left[M, \pi_{M}\right]_{G_{W}}$-type, i.e., we have an equivalence of categories

$$
\mathcal{M}: \mathcal{R}^{\left[M, \pi_{M}\right]}\left(G_{W}\right) \rightarrow \operatorname{Mod}-\mathscr{H}\left(G_{W}, \lambda_{P}\right), \quad \tau \mapsto \operatorname{Hom}_{J_{P}}\left(\lambda_{P}, \tau\right),
$$

- an injective morphism of algebras [20, (8.3), (8.4)]

$$
t_{P}: \mathscr{H}\left(M, \lambda_{M}\right) \rightarrow \mathscr{H}\left(G_{W}, \lambda_{P}\right)
$$

giving rise to the natural functor

$$
\begin{aligned}
\left(t_{P}\right)_{*}: \operatorname{Mod}-\mathscr{H}\left(M, \lambda_{M}\right) & \rightarrow \operatorname{Mod}-\mathscr{H}\left(G_{W}, \lambda_{P}\right), \\
D & \mapsto \operatorname{Hom}_{\mathscr{H}\left(M, \lambda_{M}\right)}\left(\mathscr{H}\left(G_{W}, \lambda_{P}\right), D\right),
\end{aligned}
$$

such that the following diagram commutes:

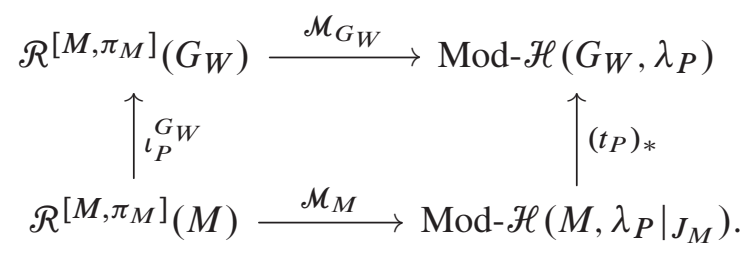

We will be interested in when the parabolic induction

$$
\tilde{\pi}|\operatorname{det}|^{s} \rtimes \pi:=\iota_{P}^{G_{W}}\left(\tilde{\pi}|\operatorname{det}|^{s} \otimes \pi\right), \quad s \in \mathbb{C},
$$

is reducible, where $\tilde{\pi}$ is a supercuspidal representation of $\tilde{G}_{Z}$ and $\pi$ is a supercuspidal representation of $G_{V}$. We stress that in the theory of covers one thinks in terms of inertial classes, hence the parameter $s$ is complex, whereas in classical references about such reducibilities, the parameter $s$ is real. In particular, by [47], if (2.6) is reducible for some $s$, which can be assumed to be real by twisting $\tilde{\pi}$ by a unitary character, then there is a real $a$ such that $\tilde{\pi}|\operatorname{det}|^{a}$ is selfdual and, assuming $\tilde{\pi}$ itself is self-dual, there is a unique real $s_{\tilde{\pi}, \pi} \geq 0$, indeed a half integer, such that (2.6) is reducible at $s= \pm s_{\tilde{\pi}, \pi}$. We keep assuming $\tilde{\pi}$ self-dual. Let $f_{\tilde{\pi}}$ be the order of the stabilizer of $\tilde{\pi}$ in the group of unramified characters of $\tilde{G}_{Z}$. By [19, Lemma 6.2.5] there are exactly two self-dual representations in the inertial class of $\tilde{\pi}$, namely $\tilde{\pi}$ and $\tilde{\pi}^{\prime}$, the twist of $\tilde{\pi}$ by the unramified character $|\operatorname{det}|^{\frac{\pi l}{f_{\tilde{\pi}} \log q}}$. The complex points of reducibility for $\tilde{\pi}$ are of the form

$$
\left\{ \pm s_{1}, \pm s_{2}+\frac{\pi i}{f_{\tilde{\pi}} \log q}\right\} \quad \text { for some } s_{1}, s_{2} \in \frac{1}{2} \mathbb{Z}_{\geq 0}
$$

and those for $\tilde{\pi}^{\prime}$ take the same form, with $s_{1}$ and $s_{2}$ exchanged. To obtain more information about these values, we will study the structures of Hecke algebras and their modules in the next section. We will also recall results of Mœglin about them in Section 2.7.

2.5. Structure of the Hecke algebras. We continue from the constructions in Section 2.4 , and briefly recall the structure of the above Hecke algebras, referring the detail to $[20,36,50]$. 
For $\tilde{G}$, if $E=F[\beta]$ is the field datum of $\mathbf{s}$, then $I_{\tilde{G}}(\tilde{\lambda})=\tilde{\mathbf{J}}$ with $\tilde{\mathbf{J}} / \tilde{J} \cong E^{\times} / U_{E} \cong\left\langle\varpi_{E}\right\rangle$, where $\varpi_{E}$ is a uniformizer of $E$. The Hecke algebra $\mathscr{H}(\tilde{G}, \tilde{\lambda})$ is isomorphic to $\mathbb{C}\left[Z, Z^{-1}\right]$, where $Z$, chosen up to a scalar, is supported on the single coset $\varpi_{E} \tilde{J}_{\Lambda}$ (note that this coset is independent of the choice of $\left.\varpi_{E}\right)$. For $G$, since $I_{G}(\lambda)=J_{\Lambda}$, we have $\mathscr{H}(G, \lambda) \cong \mathbb{C}$. Therefore, $\mathscr{H}(M, \tilde{\lambda} \otimes \lambda) \cong \mathbb{C}\left[Z, Z^{-1}\right]$.

We now fix $w$ to be either one of $\{y, z\}$ and abbreviate $\kappa_{P}=\kappa_{P}^{w}$ and $\lambda_{P}=\kappa_{P} \otimes \rho_{M}$, such that $\left.\lambda_{P}\right|_{J_{M}}=\tilde{\lambda} \otimes \lambda$. Consider the Hecke algebra $\mathscr{H}\left(G_{W}, \lambda_{P}\right)$. If $\tilde{\lambda}$ is not self-dual, then

$$
\mathscr{H}\left(G_{W}, \lambda_{P}\right) \cong \mathscr{H}(M, \tilde{\lambda} \otimes \lambda) \cong \mathbb{C}\left[Z, Z^{-1}\right] .
$$

The interesting case happens when $\tilde{\lambda}$ is self-dual, in which case it is known (see [50, Corollary 6.16] and [12, Proposition 3.3]) that

$$
\operatorname{rank}_{\mathscr{H}(M, \tilde{\lambda} \otimes \lambda)}\left(\mathscr{H}\left(G_{W}, \lambda_{P}\right)\right)=\#\left(N_{G_{W}}([M, \tilde{\pi} \otimes \pi]) / M\right)=2 .
$$

The Hecke algebra $\mathscr{H}\left(G_{W}, \lambda_{P}\right)$ has two invertible generators $T_{w}$, for $w \in\{y, z\}$. To describe them precisely, we choose two elements $s_{y}$ and $s_{z}$ (for example, we may choose $s_{1}$ and $s_{1}^{\varpi}$ in [50] or [12]), each of which is a generator for the normalizer group $N_{G_{W}}([M, \tilde{\pi} \nabla \pi])$ $\bmod M$ such that

$$
s_{y} J_{P}^{-} s_{y} \subset J_{P}^{+} \quad \text { and } \quad s_{z} J_{P}^{+} s_{z}^{-1} \subset J_{P}^{-},
$$

and moreover $\zeta:=s_{y} s_{z}=i_{M}\left(\varpi_{E} I, I\right)$ which is a $P$-positive element in the sense that

$$
\zeta J_{P}^{+} \zeta^{-1} \subset J_{P}^{+} \text {and } \zeta^{-1} J_{P}^{-} \zeta \subset J_{P}^{-} \text {. }
$$

(If we have chosen $P^{-}$instead of $P$ to define our covering type, then we have to switch $y$ and $z$.) Each of the two generators $T_{w}$, for $w \in\{y, z\}$, is supported on the single double coset $J_{P} s_{w} J_{P}$ and is defined up to a scalar. We choose the scalars so that they satisfy

$$
T_{y} * T_{z}=t_{P}(Z), \quad \text { in particular } t_{P}(Z)(\zeta)=T_{y}\left(s_{y}\right) T_{z}\left(s_{z}\right) .
$$

They also satisfy quadratic relations

$$
T_{w} * T_{w}=b_{w} T_{w}+c_{w} \mathbb{1}
$$

for certain real numbers $b_{w}$ and $c_{w}$. Here $\mathbb{1}$ is the unit in $\mathscr{H}\left(G_{W}, \lambda_{P}\right)$, which is the function supported on $J_{P}$ with $\mathbb{1}(1)=I_{\lambda_{P}}$, the identity operator on the representation space of $\lambda_{P}$.

For direct formulas for the coefficients, we follow [9, Section 1] and obtain

$$
\begin{aligned}
& c_{y}=\left(\operatorname{dim} \lambda_{P}\right)^{-1}\left[J_{P}^{+}: s_{y} J_{P}^{-} s_{y}\right] \operatorname{tr}\left(T_{y}\left(s_{y}\right) T_{y}\left(s_{y}^{-1}\right)\right), \\
& b_{y}=\left(\operatorname{dim} \lambda_{P}\right)^{-1} \sum_{u \in \frac{s_{y} J_{P}^{+} s_{y} \cap J_{P} s_{y} J_{P}}{J_{P}}} \operatorname{tr} T_{y}(u),
\end{aligned}
$$

and similarly

$$
\begin{gathered}
c_{z}=\left(\operatorname{dim} \lambda_{P}\right)^{-1}\left[s_{z} J_{P}^{-} s_{z}^{-1}: J_{P}^{+}\right] \operatorname{tr}\left(T_{z}\left(s_{z}\right) T_{z}\left(s_{z}^{-1}\right)\right), \\
b_{z}=\left(\operatorname{dim} \lambda_{P}\right)^{-1} \sum_{u \in \frac{s_{z} J_{P}^{-} s_{z}^{-1} \cap J_{P} s_{z} J_{P}}{J_{P}^{+}}} \operatorname{tr} T_{z}(u) .
\end{gathered}
$$


Later in (2.17) we will choose $s_{w}$ such that $s_{w}^{2} \in J_{M}$, so that we may normalize each $T_{w}$, up to a sign, such that

$$
T_{w}\left(s_{w}\right)^{2}=\lambda_{P}\left(s_{w}^{2}\right)
$$

which is equivalent to requiring that

$$
T_{w}\left(s_{w}\right) T_{w}\left(s_{w}^{-1}\right)=I_{\lambda_{P}}
$$

and so that $c_{y}=\left[J_{P}^{+}: s_{y} J_{P}^{-} s_{y}\right]$ and $c_{z}=\left[s_{z} J_{P}^{-} s_{z}^{-1}: J_{P}^{+}\right]$are both positive.

Indeed, by [50, Section 7.1], each $T_{w}$ comes from the generator of $\mathscr{H}\left(U_{\mathfrak{M}^{w}, E}, \tilde{\rho} \otimes \rho\right)$ which is then reduced to a Hecke algebra for the finite reductive quotient (not necessarily connected)

$$
\mathrm{G}_{w}=U_{\mathfrak{M}^{w}, E} / U_{\mathfrak{M}^{w}, E}^{1} .
$$

By Lusztig, under a suitable normalization of the generator that we will denote by $\mathcal{T}_{w}$ to avoid confusion, the quadratic relation can be written as

$$
\left(\mathcal{T}_{w}+\mathbb{1}\right) *\left(\mathcal{T}_{w}-q_{E}^{r_{w}} \mathbb{1}\right)=0
$$

for certain integers $r_{w} \geq 0$. The values of $r_{w}$ can be determined by the method in [13,34] combined with Lusztig's classification [35]. In our present situation, we can also compute these values by comparing the coefficients, or more precisely the eigenvalues, between (2.13) and (2.9), which will be done in Section 4.

2.6. Modules over Hecke algebras. It is worthwhile to mention a result, in (2.19) below, from [12] about the real parts of the points of reducibility, i.e., the values of $s_{1}$ and $s_{2}$ in (2.7), which are enough to determine the inertial Jordan blocks (cf. [13]) of a supercuspidal $\pi$ of $G$ with type $\lambda$. The result will be recalled after some prerequisites on modules over Hecke algebras.

Suppose that $\tilde{\pi}=\operatorname{cInd}_{\tilde{\mathbf{J}}} \tilde{\boldsymbol{\lambda}} \tilde{\lambda}$, where $\tilde{\lambda}$ extends to $\tilde{\mathbf{J}}$ the representation $\tilde{\lambda}$ of $\tilde{J}$. We abbreviate $\mathscr{H}_{\tilde{G}}=\mathscr{H}(\tilde{G}, \tilde{\lambda})$ and recall the equivalence of categories

$$
\mathcal{M}_{\tilde{G}}: \mathcal{R}^{[\tilde{G}, \tilde{\pi}]}(\tilde{G}) \rightarrow \operatorname{Mod}-\mathscr{H}(\tilde{G}, \tilde{\lambda}), \quad \tilde{\tau} \mapsto \hat{\tilde{\tau}}:=\operatorname{Hom}_{\tilde{J}}(\tilde{\lambda}, \tilde{\tau}),
$$

where the $\mathscr{H}_{\tilde{G}}$-action on $\hat{\tilde{\tau}}$ is given by

$$
(\phi \cdot f)(w)=\int_{\tilde{G}} \tilde{\tau}\left(g^{-1}\right) \circ \phi \circ f(g)(w) d g, \quad \phi \in \hat{\tilde{\tau}}, f \in \mathscr{H}_{\tilde{G}}, w \in V_{\tilde{\lambda}} .
$$

(Here $V_{\tilde{\lambda}}$ is the representation space of $\tilde{\lambda}$, and recall that we fixed the Haar measure on $\tilde{G}$ such that the measure of $\tilde{J}_{\Lambda}$ is 1.) In particular, we have

$$
\phi \cdot Z=\tilde{\tau}\left(\varpi_{E}^{-1}\right) \circ \phi \circ Z\left(\varpi_{E}\right) .
$$

The group of unramified characters of $\tilde{G}$ acts on $\mathcal{R}^{[\tilde{G}, \tilde{\pi}]}(\tilde{G})$ by

$$
\tilde{\chi} \cdot \tilde{\tau}=\tilde{\tau} \otimes \tilde{\chi} .
$$

and on $\mathscr{H}_{\tilde{G}}$ by

$$
(\tilde{\chi} \cdot f)(x)=\tilde{\chi}(x) f(x), \quad f \in \mathscr{H}_{\tilde{G}}, x \in \tilde{G},
$$

which induces an action on Mod- $\mathscr{H}_{\tilde{G}}$ naturally. The equivalence map $\mathcal{M}_{\tilde{G}}$ is hence equivariant under this action. 
We now turn to our constructed type $\lambda_{P}$ in $G_{W}$ and abbreviate $\mathscr{H}_{M}=\mathscr{H}\left(M,\left.\lambda_{P}\right|_{J_{M}}\right)$ and $\mathscr{H}_{G_{W}}=\mathscr{H}\left(G_{W}, \lambda_{P}\right)$. For $s \in \mathbb{C}$, we denote by $D_{s}$ the simple right $\mathscr{H}_{M}$-module

$$
\mathcal{M}_{M}\left(\tilde{\pi}|\operatorname{det}|^{s} \otimes \pi\right)=\operatorname{Hom}_{J_{M}}\left(\lambda_{M}, \tilde{\pi}|\operatorname{det}|^{S} \otimes \pi\right),
$$

necessarily one-dimensional. We then denote by $X_{S}$ the right $\mathscr{H}_{G}$-module

$$
\mathcal{M}\left(\tilde{\pi}|\operatorname{det}|^{S} \rtimes \pi\right)=\operatorname{Hom}_{J_{P}}\left(\lambda_{P}, \tilde{\pi}|\operatorname{det}|^{S} \rtimes \pi\right) \cong \operatorname{Hom}_{\mathscr{H}_{M}}\left(\mathscr{H}_{G}, D_{S}\right),
$$

where the last isomorphism is given by the commutative diagram (2.5).

On the other hand, the morphism $t_{P}$ that renders diagram (2.5) commutative satisfies

$$
t_{P}(Z)(\zeta)=\Delta_{P}(\zeta)^{-1 / 2} Z\left(\varpi_{E}\right)
$$

where $\Delta_{P}$ is the modular character of $P$ and $\zeta=s_{y} s_{z}=i_{M}\left(\varpi_{E} I, I\right)$ as in the previous subsection.

By (2.14), the scalar action of $Z$ on $D_{s}$ is given by $q_{E}^{s} \tilde{\lambda}\left(\varpi_{E}^{-1}\right) Z\left(\varpi_{E}\right)$. Since (2.15) relates $Z\left(\varpi_{E}\right)$ and $t_{P}(Z)(\zeta)$, and the latter is given by (2.8), we get the scalar of the action of $Z$ on $D_{s}$ as

$$
q_{E}^{s} \Delta_{P}(\zeta)^{1 / 2} \tilde{\lambda}\left(\varpi_{E}^{-1}\right) T_{y}\left(s_{y}\right) T_{z}\left(s_{z}\right) .
$$

We now impose an assumption that

- the operators $\tilde{\lambda}\left(\varpi_{E}\right), T_{y}\left(s_{y}\right)$, and $T_{z}\left(s_{z}\right)$ on $V_{\tilde{\lambda}}$ have finite orders.

This assumption can be easily satisfied when $\tilde{\lambda}$ is self-dual and $s_{y}$ and $s_{z}$ are chosen to be simple enough; for example, we can and do pick

$$
s_{y}=\left[\begin{array}{lll} 
& & I \\
I & &
\end{array}\right] \text { and } s_{z}=\left[\begin{array}{lll} 
& & -\varpi_{E}^{-1} I \\
\varpi_{E} I & &
\end{array}\right],
$$

which by (2.12) means that $T_{y}\left(s_{y}\right)^{2}=1$ and $T_{z}\left(s_{z}\right)^{2}=\tilde{\lambda}(-1) \otimes \lambda(1)$. (The $I$ s at the antidiagonal corners of $s_{y}$ and $s_{z}$ are the identity matrix under the identification of $Z_{-}$and $Z_{+}$in Assumptions 2.2) We also use the fact

$$
\Delta_{P}(\zeta)=\left[J_{P}^{+}: \zeta J_{P}^{+} \zeta^{-1}\right]=\left[s_{y} J_{P}^{+} s_{y}: J_{P}^{-}\right]\left[s_{z} J_{P}^{-} s_{z}^{-1}: J_{P}^{+}\right]
$$

which is just $c_{y} c_{z}$ with our normalization which, we point out, is only up to sign so far. We write $c_{w}^{1 / 2}$ for the positive square root of $c_{w}$.

We now compare the quadratic relations (2.9) for $T_{w}$ and (2.13) for $\mathcal{T}_{w}$, a scalar multiple of $T_{w}$, and obtain, for $w \in\{y, z\}$ :

$$
b_{w}=\epsilon_{w} c_{w}^{1 / 2}\left(q_{E}^{r_{w} / 2}-q_{E}^{-r_{w} / 2}\right),
$$

where $\epsilon_{w}$ is a sign. Hence the eigenvalues of $T_{w}$ are

$$
\epsilon_{w} c_{w}^{1 / 2} q_{E}^{r_{w} / 2} \text { and }-\epsilon_{w} c_{w}^{1 / 2} q_{E}^{-r_{w} / 2}
$$

and the possible products of eigenvalues of $T_{y}$ and $T_{z}$ are

$$
\epsilon_{y} \epsilon_{z} c_{y}^{1 / 2} c_{z}^{1 / 2} q_{E}^{ \pm\left(r_{y}+r_{z}\right) / 2} \text { and }-\epsilon_{y} \epsilon_{z} c_{y}^{1 / 2} c_{z}^{1 / 2} q_{E}^{ \pm\left(r_{y}-r_{z}\right) / 2}
$$


When $\tilde{\pi}|\operatorname{det}|^{S} \rtimes \pi$, and hence $X_{S}$, is reducible, the eigenvalue of $t_{P}(Z)$ on a one-dimensional submodule is a product of one of those of $T_{y}$ and one of those of $T_{z}$. By comparing the absolute values of (2.16) and (2.18), we obtain

$$
\left\{s_{1}, s_{2}\right\}=\left\{\frac{r_{y}+r_{z}}{2}, \frac{\left|r_{y}-r_{z}\right|}{2}\right\} .
$$

In Section 4, we will provide more detail about the products of eigenvalues, and obtain the conditions on the cuspidal types for which reducibilities happen. In particular, we will determine the signs $\epsilon_{y}$ and $\epsilon_{z}$ under the above setup, deriving them from (2.10) and (2.11).

2.7. Mœglin's results. In this subsection, we provide two results due to Mœglin, for which we require the characteristic of $F$ to be 0 . The first one is called a finiteness result in [38, 4. Proposition], and is improved in [39, Theorem 3.2.1]. The second one is a parity result on Langlands parameters [38, 5.6. Proposition]. See also [40, 8.3.5] for non-quasi-split groups.

Given a supercuspidal representation $\pi$ of a unitary group $G=G_{V}$, then

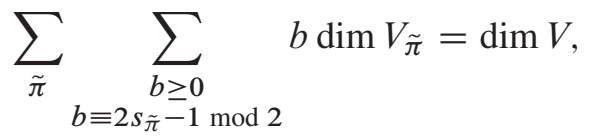

where the sum $\sum_{\tilde{\pi}}$ ranges over all supercuspidal representations $\tilde{\pi}$, each of which is a supercuspidal representation of a general linear group $\tilde{G}_{V_{\tilde{\pi}}}$ for some space $V_{\tilde{\pi}}$, such that $\tilde{\pi}|\operatorname{det}|^{S} \rtimes \pi$ is reducible at $s_{\tilde{\pi}} \in \frac{1}{2} \mathbb{Z}$ with $s_{\tilde{\pi}} \geq 1$. In particular, in the extreme case (which is studied in this paper), if there exists such a $\tilde{\pi}$ with $\operatorname{dim} V_{\tilde{\pi}}=\operatorname{dim} V$, then it is the unique such representation that gives rise to the reducibility above with $s_{\tilde{\pi}}=1$, in which case $\tilde{\pi}$ is the base change of $\pi$.

We now switch to the Galois side and look at Langlands parameters. We recall that $c$ is the generator of $\operatorname{Gal}\left(F / F_{\bullet}\right)$. We call an (semisimple, smooth) irreducible representation $\tilde{\varphi}$ of $\mathcal{W}_{F}$ (conjugate-)self-dual if $\tilde{\varphi} \cong{ }^{\sigma} \tilde{\varphi}:={ }^{c} \tilde{\varphi}^{\vee}$, where

$$
{ }^{c} \tilde{\varphi}(w)=\tilde{\varphi}\left(c^{-1} w c\right) \text { for all } w \in \mathcal{W}_{F},
$$

and $\tilde{\varphi}^{\vee}$ is the contragredient of $\tilde{\varphi}$. Hence an irreducible representation $\tilde{\varphi}$ is self-dual if there exists a non-degenerate bilinear form $B$ on $\mathbf{V}:=\mathbb{C}^{\operatorname{deg} \tilde{\varphi}}$ such that

$$
B\left({ }^{c} \tilde{\varphi}(w) u, \tilde{\varphi}(w) v\right)=B(u, v) \quad \text { for all } u, v \in \mathbf{V} \text { and } w \in \mathcal{W}_{F} .
$$

We also define a parity on $\tilde{\varphi}$ : call $\tilde{\varphi}$ conjugate-orthogonal (resp. conjugate-symplectic) if, furthermore,

$$
B(u, v)=\operatorname{sgn}(\tilde{\varphi}) B\left(\tilde{\varphi}\left(c^{2}\right) v, u\right) \quad \text { for all } u, v \in \mathbf{V},
$$

where $\operatorname{sgn}(\tilde{\varphi})=1$ (resp. -1$)$. The parity of $\tilde{\varphi}$, as an irreducible representation of $\mathcal{W}_{F}$, can also be defined using Asai L-functions: if $r_{A}$ is the Asai representation of GL(V) (see [37, A.2.1]), then $\tilde{\varphi}$ is conjugate-orthogonal if and only if

$$
L\left(s, \tilde{\varphi}, r_{A}\right):=L\left(s, r_{A} \circ \tilde{\varphi}\right), \quad s \in \mathbb{C},
$$

has a pole at $s=0$. 


\section{Ramified unitary groups}

In this section, we will impose a condition on our inducing types (see Definition 3.3), the "strongly ramified" condition. The reason of imposing this condition was explained at the end of the introduction section. We will see that this condition forces our unitary group to be ramified, i.e., $F / F_{\bullet}$ is ramified, and our inducing types take a simple form. With these types, we will state our results on the values of the coefficients $b_{w}$ for $w \in\{y, z\}$, the points of reducibility in Section 3.2, and the base change of supercuspidal representations in Section 3.3. The calculations will be postponed to Section 4.

3.1. The strongly ramified case. We first return to the setup in Section 2.2 . To proceed, let $\mathbf{s}=[\Lambda, r, 0, \beta]$ be a simple stratum, and denote $E=F[\beta]$. Together with $\mathbf{s}$ is a sequence of approximating simple strata $\mathbf{s}_{j}:=\left[\tilde{\mathfrak{U}}, r, r_{j}, \gamma_{j+1}\right]$, for $j=0, \ldots, d$, satisfying the conditions in $[19,(2.4 .2)]$; in particular,

(i) the simple stratum $\mathbf{s}_{j}$ is equivalent to [ $\left.\tilde{\mathfrak{U}}, r, r_{j}, \gamma_{j}\right]$,

(ii) if we denote by

- $\tilde{B}_{j}$ the centralizer of $\gamma_{j}$ in $\tilde{A}$,

- $\tilde{\mathfrak{B}}_{j}=\tilde{B}_{j} \cap \tilde{\mathfrak{A}}$, and

- $s_{j}$ the tame corestriction of $A$ relative to $F\left[\gamma_{j}\right] / F$,

then the derived stratum $\left[\tilde{\mathfrak{B}}_{j+1}, r_{j}, r_{j}-1, s_{j+1}\left(\gamma_{j}-\gamma_{j+1}\right)\right]$ is equivalent to a simple stratum, say $\left[\tilde{\mathfrak{B}}_{j+1}, r_{j}, r_{j}-1, \delta_{j}\right]$.

The numbers $\left\{r_{j}\right\}_{j=0}^{d}$ are the critical exponents of $\mathbf{s}$. For the moment we do not require the definition, but the simplicity of $\left[\tilde{\mathfrak{B}}_{j+1}, r_{j}, r_{j}-1, \delta_{j}\right]$ implies that $v_{\tilde{\mathfrak{B}}_{j+1}}\left(\delta_{j}\right)=-r_{j}$.

We now assume that $\mathbf{s}$ is skew, we can apply $[48,(1.10)]$ and do choose all $\mathbf{s}_{j}$ to be also skew. If we also choose the tame corestriction $s_{j}$ to be $\alpha$-equivariant, then we can also assume $\left[\tilde{\mathfrak{B}}_{j+1}, r_{j}, r_{j}-1, \delta_{j}\right]$ to be skew. Let $E_{\bullet}$ be the subfield of $E$ fixed by $c=-\alpha$. We now impose the following condition on the field extension $E / E_{\bullet}$ :

$$
E / E_{\bullet} \text { is a quadratic ramified extension. }
$$

This condition implies that $e=e(E / F)$ is odd. If $K$ is an intermediate subfield between $E$ and $F$, and $K_{\bullet}$ is the fixed-field of $K$ by the involution $-\alpha$, then $K / K_{\bullet}$ is also quadratic ramified. In particular, our unitary group is forced to be ramified.

Proposition 3.1. When $E / E_{\bullet}$ is ramified, all $\left\{r_{j}\right\}_{j=1}^{d}$ are odd.

Proof. Let $\left[\tilde{\mathfrak{B}}_{j+1}, r_{j}, r_{j}-1, \delta_{j}\right]$ be the simple stratum equivalent to the derived stratum $\left[\tilde{\mathfrak{B}}_{j+1}, r_{j}, r_{j}-1, s_{j+1}\left(\gamma_{j}-\gamma_{j+1}\right)\right]$, which is assumed to be skew; in particular, we have $\delta_{j}={ }^{\alpha} \delta_{j}$. Hence $-\alpha$ defines an involution on the field $F\left[\delta_{j}\right]$ whose restriction to $F$ is $c$, the Galois conjugation of $F / F_{\bullet}$. We denote its fixed field by $F\left[\delta_{j}\right]_{\bullet}$. By [19, (2.2.8)] and since $e(E / F)$ is odd, $\left.e\left(F\left[\delta_{j}\right] / F\right]\right)$ is also odd. This implies that $F\left[\delta_{j}\right] / F\left[\delta_{j}\right] \bullet$ is ramified. The $\alpha$-invariance of $\delta_{j}$ implies that $v_{F\left[\delta_{j}\right]}\left(\delta_{j}\right)$ is odd, and so is

$$
r_{j}=-v_{F\left[\delta_{j}\right]}\left(\delta_{j}\right) e(E / F) / e\left(F\left[\delta_{j}\right] / F\right) .
$$


This proposition facilitates our calculations remarkably. First of all, we immediately see that $\tilde{J}^{1}=\tilde{H}^{1}$ from their constructions [19, Section 3.1]. We also have $\tilde{J}_{\mathfrak{M}^{y}}^{1}=\tilde{H}_{\mathfrak{M}}^{1} \tilde{H}^{1}$ since $\mathfrak{M}^{y}$ is a dilation and shift of a lattice sequence equivalent to $\Lambda$, and as for $\tilde{J}_{\mathfrak{m}}^{1}=\tilde{H}_{\mathfrak{m}}^{1}$ we just note that the critical exponents of $(\beta, \beta, \beta)$ are $\left\{3 r_{j}\right\}_{j=1}^{d}$. The Heisenberg representation containing a simple character $\tilde{\theta}$ is just $\tilde{\theta}$ itself, and so any beta-extension of $\tilde{\theta}$ is a character. If moreover $\tilde{\theta}$ is self-dual, which is assumed from now on, then the $p$-primary beta extension $\tilde{\kappa}_{0}$ admits further self-dual extensions to $\tilde{\mathbf{J}}$. We can list them by first fixing uniformizers $\varpi_{F}$ and $\varpi_{E}$ for $F$ and $E$ respectively, whose squares belong to $F_{\bullet}$ and $E_{\bullet}$.

Proposition 3.2. There are two choices of self-dual extensions $\tilde{\boldsymbol{\kappa}}$ of $\tilde{\kappa}_{0}$, given by

$$
\tilde{\boldsymbol{\kappa}}\left(\varpi_{F}\right)= \pm 1 \text { and } \tilde{\boldsymbol{\kappa}}\left(\varpi_{F}\right)=\tilde{\boldsymbol{\kappa}}\left(\varpi_{E}\right) .
$$

Proof. The $p$-primary condition on $\tilde{\kappa}_{0}$ implies that it is self-dual and $\left.\tilde{\boldsymbol{\kappa}}\right|_{\boldsymbol{\mu}_{E \bullet}}=1$. With the choice of $\varpi_{F}$, we have ${ }^{c} \varpi_{F}=-\varpi_{F}$, and so $\tilde{\kappa}\left(\varpi_{F}\right)^{2}=\tilde{\kappa}(-1)=1$. Also ${ }^{c} \varpi_{E}=-\varpi_{E}$, so a similar argument shows $\tilde{\kappa}\left(\varpi_{E}\right)^{2}=1$. To show the last equality, we write $\varpi_{E}^{e}=\varpi_{F} z u$ for some $z \in \mu_{E}=\mu_{E_{\bullet}}$ and $u \in U_{E}^{1}$. The facts that ${ }^{c} \varpi_{E}=-\varpi_{E}$ and $e$ is odd imply that $u \in U_{E}^{1}$. Since $\tilde{\theta}$ is self-dual and has order a power of $p$, we have $\tilde{\kappa}(u)=\tilde{\theta}(u)=1$. We now have $\tilde{\kappa}\left(\varpi_{E}\right)^{e}=\tilde{\kappa}\left(\varpi_{F}\right)$. Since $e$ is odd, combining with $\tilde{\kappa}\left(\varpi_{F}\right)^{2}=\tilde{\kappa}\left(\varpi_{E}\right)^{2}=1$, we have $\tilde{\kappa}\left(\varpi_{F}\right)=\tilde{\kappa}\left(\varpi_{E}\right)$. This completes the proof.

For convenience, we pick the extension $\tilde{\boldsymbol{\kappa}}=\tilde{\kappa}_{0}$ such that $\tilde{\kappa}_{0}\left(\varpi_{E}\right)=1$ from now on. Finally, we choose our covering type $\lambda_{P}=\kappa_{P} \otimes \rho_{M}$ such that

$$
\kappa_{P}=\kappa_{P}^{y},
$$

since in this case $\left.\kappa_{P}\right|_{J_{M}}=\tilde{\kappa}_{0} \otimes \kappa_{0}$, i.e., it is $p$-primary.

We also take a self-dual level-zero type $\tilde{\boldsymbol{\rho}}$ of $\tilde{\boldsymbol{J}}=E^{\times} \tilde{\boldsymbol{J}}$, i.e., it is an extension of a representation $\tilde{\rho}$ of $\tilde{J}$ inflated from an irreducible cuspidal representation of $\tilde{J} / \tilde{J}^{1} \cong \mathrm{GL}_{f_{0}}\left(\mathbf{k}_{E}\right)$, where $f_{0}=n /[E: F]$. Denote by $E^{\prime}$ the unramified extension of $E$ of degree $f_{0}$ in $\tilde{A}$, so that $E^{\prime \times}$ is embedded into $\tilde{G}$ accordingly. Associated to $\tilde{\boldsymbol{\rho}}$ is a level-zero character $\tilde{\xi}:=\tilde{\xi}_{\tilde{\boldsymbol{\rho}}}$ of $E^{\prime \times}$ which is $\operatorname{Gal}\left(E^{\prime} / E\right)$-regular (which is equivalent to say that $\left.\tilde{\xi}\right|_{U_{E^{\prime}}}$ is $\operatorname{Gal}\left(E^{\prime} / E\right)$-regular), such that $\tilde{\boldsymbol{\rho}}$ is self-dual if and only if $\tilde{\xi}$ is, in which case it implies that either $f_{0}=1$ or else, by [43, Lemmas 2.1 and 5.1], the conjugation $-\alpha$ on $E$ extends to $E^{\prime}$ which is unramified over the fixed field $E_{\bullet}^{\prime}=\left(E^{\prime}\right)^{-\alpha}$, and in particular $f_{0}$ is even.

We postpone the discussion of the case $f_{0}>1$ to Section 3.4 and proceed to our main results under the condition $f_{0}=1$. The reason of imposing this condition is accounted for in Proposition 3.13: if $f_{0}>1$, the two candidates for the base change do not have the same parity, therefore the decision between them can be made by computing Asai L-functions, as was briefly explained in the last paragraph of the introduction.

We hence look at the case when

$$
f_{0}=\operatorname{dim}_{F} V /[E: F]=1,
$$

so that $\tilde{\boldsymbol{\rho}}=\tilde{\xi}_{\tilde{\boldsymbol{\rho}}}$, i.e., $\tilde{\boldsymbol{\rho}}$ is a self-dual character. In this case,

$$
\tilde{\rho}=\left.\tilde{\rho}\right|_{\mu_{E}} \text { is at most quadratic and } \tilde{\rho}\left(\varpi_{E}\right)^{2}=\tilde{\rho}(-1),
$$

so that $\tilde{\rho}\left(\varpi_{E}\right)$ is a fourth root of unity. 
Let $\tilde{\lambda}$ be an extended maximal simple type of the form $\tilde{\kappa}_{0} \otimes \tilde{\rho}$, where $\tilde{\boldsymbol{\kappa}}_{0}$ is the unique extension of a self-dual simple character given by Proposition 3.2 with $\tilde{\boldsymbol{\kappa}}_{0}\left(\varpi_{E}\right)=1$, and $\tilde{\boldsymbol{\rho}}$ is a self-dual level-zero type. We summarize the above conditions in the following terminology.

Definition 3.3. We call the extended type $\tilde{\lambda}$ for $\tilde{G}$, as well as its induced supercuspidal representation $\tilde{\pi}$, strongly ramified when both (3.1) and (3.2) are satisfied, and similarly for $\lambda$ and $\pi$ for $G$.

Finally, we recall from [35, Section 5] (or see [34, Section 7.6]) the values of the integers $r_{w}$, with $w \in\{y, z\}$, that appear in the quadratic relations in (2.13) at the end of Section 2.5. From our construction we have

$$
\mathrm{G}_{y} \cong \mathrm{O}_{3, \mathbf{k}_{E}}, \quad \text { so that } r_{y}=1,
$$

and also

$$
\mathrm{G}_{z} \cong \mathrm{Sp}_{2, \mathbf{k}_{E}} \times \mathrm{O}_{1, \mathbf{k}_{E}}, \quad \text { so that } r_{z}= \begin{cases}1 & \text { if } \tilde{\rho} \text { is trivial, } \\ 0 & \text { otherwise. }\end{cases}
$$

From (2.19) the positive real parts of the points of reducibility are

$$
\{0,1\} \text { if } \tilde{\rho} \text { is trivial, and }\{1 / 2,1 / 2\} \text { otherwise. }
$$

There is a support-preserving algebra morphism from [50, (7.3)],

$$
\mathscr{H}\left(U_{\mathfrak{M}^{w}, E}, \tilde{\rho} \otimes \rho\right) \hookrightarrow \mathscr{H}\left(G_{W}, \lambda_{P}\right) \quad \text { for each } w \in\{y, z\} .
$$

Hence if we view each generator $T_{w}$ as an endomorphism of the module $X_{s}$ corresponding to the parabolic induction $\tilde{\pi}|\operatorname{det}|^{S} \rtimes \pi$, then the above results, combining with the quadratic relation (2.9), imply that

$$
b_{w}=\epsilon_{w}\left(q_{E}-1\right)\left(c_{w} / q_{E}\right)^{1 / 2},
$$

where $\epsilon_{w}$ is a sign. We will provide the precise value for the sign $\epsilon_{w}$ in the next subsection, with calculation given in Section 4.

3.2. Reducibility results. To state the main result on the eigenvalues for the quadratic relations, we impose the following assumptions which are used in Section 4.

- We take a strongly ramified supercuspidal representation $\pi$ of $G$, i.e., if $(\theta, \rho)$ is a pair consisting of a simple character and a level zero cuspidal representation defining $\pi$, then that $E / E_{\bullet}$ is ramified and $\operatorname{dim} V /[E: F]=1$.

- The extension $E / F$ is tamely ramified, which allows us to assume that $E_{j}=F\left[\gamma_{j}\right]$ is contained in $E_{j-1}=F\left[\gamma_{j-1}\right]$ for all $j$, forming a tower of intermediate extensions between $E$ and $F$.

We also take a supercuspidal representation $\tilde{\pi}$ of $\tilde{G}$ constructed by $(\tilde{\theta}, \tilde{\boldsymbol{\rho}})$, with $\theta=\left(\left.\tilde{\theta}\right|_{H^{1}}\right)^{1 / 2}$, satisfying the same conditions above, i.e., $\tilde{\pi}$ is strongly ramified, and $E / F$ is tamely ramified. The following theorem will be proven in Section 4:

Theorem 3.4. Suppose that $\tilde{\pi}$ and $\pi$ satisfy the above conditions.

(i) The coefficient $b_{y}$ of the quadratic relation of $T_{y}$ is

$$
b_{y}=\tilde{\rho}(-2) \rho(-1) T_{y}\left(s_{y}\right)\left(q_{E}-1\right)\left(c_{y} / q_{E}\right)^{1 / 2} .
$$


(ii) The coefficient $b_{z}$ of the quadratic relation of $T_{z}$ is given as follows.

(a) When $\left.\tilde{\rho}\right|_{\mu_{E}}=\left(\frac{\dot{\mu_{E}}}{\boldsymbol{\mu}}\right) f(E / F)$, then $b_{z}=0$.

(b) When $\left.\tilde{\rho}\right|_{\boldsymbol{\mu}_{E}}=\left(\frac{\dot{\cdot}}{\boldsymbol{\mu}_{E}}\right) f(E / F)-1$, then

$$
b_{z}=\tilde{\rho}(-2) \epsilon_{z}^{P}\left(\varpi_{E}, \mathbf{s}\right) T_{z}\left(s_{z}\right)\left(q_{E}-1\right)\left(c_{z} / q_{E}\right)^{1 / 2},
$$

where $\epsilon_{z}^{P}\left(\varpi_{E}, \mathbf{s}\right)$ is a sign, associated to a quadratic Gauss sum and determined by the choice of $\varpi_{E}$ and the simple stratum $\mathbf{s}$.

(The exact value of $\epsilon_{z}^{P}\left(\varpi_{E}, \mathbf{s}\right)$ is given in (4.20).)

For the purpose of base change, the case when $\left.\tilde{\rho}\right|_{\mu_{E}}=\left(\frac{\cdot}{\mu_{E}}\right) f(E / F)$ is unimportant.

Corollary 3.5. The following statements hold.

(i) The two eigenvalues of $T_{y}$ are

$$
-\tilde{\rho}(-2) \rho(-1) T_{y}\left(s_{y}\right)\left(c_{y} / q_{E}\right)^{1 / 2} \text { and } \tilde{\rho}(-2) \rho(-1) T_{y}\left(s_{y}\right)\left(c_{y} / q_{E}\right)^{1 / 2} q_{E} .
$$

(ii) When $\left.\tilde{\rho}\right|_{\mu_{E}}=\left(\frac{\cdot}{\mu_{E}}\right)^{f(E / F)-1}$, the two eigenvalues of $T_{z}$ are

$$
-\tilde{\rho}(-2) \epsilon_{z}^{P}\left(\varpi_{E}, \mathbf{s}\right) T_{z}\left(s_{z}\right)\left(c_{z} / q_{E}\right)^{1 / 2} \text { and } \tilde{\rho}(-2) \epsilon_{z}^{P}\left(\varpi_{E}, \mathbf{s}\right) T_{z}\left(s_{z}\right)\left(c_{z} / q_{E}\right)^{1 / 2} q_{E} \text {. }
$$

We now choose $\tilde{\pi}=\tilde{\pi}(\tilde{\theta}, \tilde{\boldsymbol{\rho}})$ with

$$
\left.\tilde{\rho}\right|_{\mu_{E}}=\left(\frac{\cdot}{\mu_{E}}\right)^{f(E / F)-1} \text { and } \tilde{\rho}\left(\varpi_{E}\right)=\rho(-1) \epsilon_{z}^{P}\left(\varpi_{E}, \mathbf{s}\right) .
$$

This leads to the following desired result (see the next remark for the other choice of $\left.\tilde{\rho}\right|_{\boldsymbol{\mu}_{E}}$ ).

Corollary 3.6. The points of reducibility of $\tilde{\pi}|\operatorname{det}|^{S} \rtimes \pi$ are

$$
\pm 1 \text { and } \frac{\pi i}{\log q_{E}} \text {. }
$$

Proof. Since the eigenvalue of $t_{P}(Z)$ is the product of eigenvalues of $T_{y}$ and $T_{z}$, the comparison in (2.16) gives

$$
\tilde{\boldsymbol{\rho}}\left(\varpi_{E}^{-1}\right) q_{E}^{s}=\epsilon_{z}^{P}\left(\varpi_{E}, \mathbf{s}\right) \rho(-1) \cdot\left(-1 \text { or } q_{E}^{ \pm 1}\right) .
$$

The corollary follows by solving $s$.

Remark 3.7. If $\left.\tilde{\rho}\right|_{\boldsymbol{\mu}_{E}}=\left(\frac{\dot{\boldsymbol{\mu}_{E}}}{)} f(E / F)\right.$, then we put $\tilde{\boldsymbol{\rho}}\left(\varpi_{E}^{-1}\right)= \pm \rho(-1) \tilde{\rho}(-2) \tilde{\rho}(-1)^{1 / 2}$ and use similar arguments as above to show that points of reducibility are

$$
\pm \frac{1}{2} \quad \text { and } \quad \pm \frac{1}{2}+\frac{\pi i}{\log q_{E}}
$$

The choice of the square root $\tilde{\rho}(-1)^{1 / 2}$ for $\tilde{\rho}\left(\varpi_{E}\right)$ does not matter as we will discard this case for base change anyway.

The following remark explains Corollaries 3.5 and 3.6 are independent of the various choices made throughout the progress. 
Remark 3.8. Note that both $T_{w}\left(s_{w}\right)$, for $w \in\{y, z\}$, have been cancelled out in (3.4). Indeed the reducibility points, as well as the base change result in Theorem 3.9 below, are independent of the normalizations of $T_{w}$ (and also that of $Z$ ) and the choices of $s_{w}$. Moreover, as explained in Section 2.5, if we have chosen $P^{-}$instead of $P$, then we need to switch the roles of $y$ and $z$. In this case, $\epsilon_{z}^{P}\left(\varpi_{E}, \mathbf{s}\right)$ should then be denoted by $\epsilon_{y}^{P^{-}}\left(\varpi_{E}, \mathbf{s}\right)$. Our corollaries are clearly independent of choosing $P$ or $P^{-}$.

3.3. The main result for base change. Let us first look at a simple case when the representations are characters, in which case $E=F$. The base change $\tilde{\rho}$ of a character $\rho$ from $\mathrm{U}_{1}\left(F / F_{\bullet}\right)$ to $\mathrm{GL}_{1}(F)$ is

$$
\tilde{\rho}(x)=\rho\left(x^{\sigma} x\right) \quad \text { for } x \in F^{\times}
$$

or more explicitly,

$$
\left.\rho\right|_{\left(U_{F}^{1}\right)^{\sigma}}=\left(\left.\tilde{\rho}\right|_{\left(U_{F}^{1}\right)^{\sigma}}\right)^{1 / 2},\left.\quad \tilde{\rho}\right|_{\mu_{F}} \equiv 1, \quad \text { and } \quad \tilde{\rho}\left(\varpi_{F}\right)=\rho(-1) .
$$

Note that the restriction $\left.\tilde{\rho}\right|_{\boldsymbol{\mu}_{F}}$ has no relation with the level-zero part of $\rho$, i.e., the character of the finite reductive quotient of $\mathrm{U}_{1}$, which is $\mathrm{O}_{1}=\{ \pm 1\}$. However, if we change $\tilde{\rho}$ by twisting a quadratic unramified character, then $\rho(-1)$ is changed to another sign.

We now consider the strongly ramified supercuspidal representations $\pi=\pi(\theta, \rho)$ and $\tilde{\pi}=\tilde{\pi}(\tilde{\theta}, \tilde{\boldsymbol{\rho}})$ as in Definition 3.3. The following theorem, the main result of our paper, provides the relations between $(\theta, \rho)$ and $(\tilde{\theta}, \tilde{\boldsymbol{\rho}})$ for which $\tilde{\pi}$ is the base change of $\pi$.

Theorem 3.9. Suppose that $\operatorname{char}(F)=0$, and the simple characters $\theta$ and $\tilde{\theta}$ and the tamely ramified characters $\tilde{\boldsymbol{\rho}}$ and $\rho$ are related as follows.

(i) $\theta=\left(\left.\tilde{\theta}\right|_{H^{1}}\right)^{1 / 2}$,

(ii) $\left.\tilde{\rho}\right|_{\boldsymbol{\mu}_{E}}=\left(\frac{\dot{\mu_{E}}}{)} f(E / F)-1\right.$, and

(iii) $\tilde{\boldsymbol{\rho}}\left(\varpi_{E}\right)=\rho(-1) \epsilon_{z}^{P}\left(\varpi_{E}, \mathbf{s}\right)$, where $\epsilon_{z}^{P}\left(\varpi_{E}, \mathbf{s}\right)$ is the sign appearing in Theorem 3.4.

Then $\tilde{\pi}$ is the base change of $\pi$.

This can easily be deduced from the reducibility result in the previous subsection, together with the finiteness result of Mœglin (2.20). One may notice that (3.5) is a special case of the theorem.

Remark 3.10. We will prove in Proposition 4.8 that relation (iii) in Theorem 3.9 is independent of the choice of the uniformizer $\varpi_{E}$.

Remark 3.11. By $[38,7.1]$, if in general a discrete series parameter, when viewed as a representation of the Weil-Deligne group, has $k$ irreducible components, then its corresponding L-packet contains $2^{k-1}$ members. In our case when the base change representation is supercuspidal, then its parameter is an irreducible representation, and the L-packet is a singleton.

3.4. Appendix: The strongly ramified case and parity. This appendix is a sequel to Section 3.1, and does not intervene with our main results. We do not assume that $F / F_{\bullet}$ is ramified. 
We provide a result on the parity of a (conjugate-)self-dual supercuspidal representation. To this end, we have to switch to the Galois side via the local Langlands correspondence for $\mathrm{GL}_{n}$, and assume that $\operatorname{char}(F)=0$. We call a self-dual supercuspidal representation $\tilde{\pi}$ of a general linear group conjugate-orthogonal (resp. conjugate-symplectic) if the Asai L-function [46] for $\tilde{\pi}$,

$$
L\left(s, \tilde{\pi}, r_{A}\right), \quad s \in \mathbb{C},
$$

has a pole at $s=0$. By [25], we know that $\tilde{\pi}$ is conjugate-orthogonal (resp. conjugate-symplectic) if its Langlands parameter is so (see (2.21) and (2.22)).

Remark 3.12. A (conjugate-)self-dual character $\tilde{\chi}$ of $\mathrm{GL}_{1}(F)$ (i.e., ${ }^{\sigma} \tilde{\chi}=\tilde{\chi}$ ) is conjugate-orthogonal (resp. conjugate-symplectic) if and only if $\left.\tilde{\chi}\right|_{F_{0} \times}$ is trivial (resp. is equal to $\delta_{F / F_{\bullet}}$, the character on $F_{\bullet}{ }^{\times}$with kernel the norm group $N_{F / F_{\bullet}}\left(F^{\times}\right)$). In particular, the quadratic unramified character $|\cdot|^{\pi i / \log q}$ is conjugate-orthogonal (resp. conjugate-symplectic) when $F / F_{\bullet}$ is ramified (resp. unramified).

Let $\tilde{\pi}$ be a supercuspidal representation of the group $\tilde{G}_{V} \cong \mathrm{GL}_{n}(F)$, containing a simple character with underlying stratum $\mathbf{s}=[\Lambda, r, 0, \beta]$. Denote the field datum $E=F[\beta]$ and put $f_{0}=n /[E: F]$. Then $f_{\tilde{\pi}}$ in $(2.7)$ is equal to $f_{0} f(E / F)$. Similar to the construction discussed in Section 3.1, let $E^{\prime} / E$ be an unramified extension of degree $f_{0}$, such that the associated level-zero component corresponds to a $\operatorname{Gal}\left(E^{\prime} / E\right)$-regular character of $E^{\prime \times}$. Suppose now $\tilde{\pi}$ is self-dual, and $-\alpha$ restricts to a conjugation on $E$ extending $c$ on $F$. The conjugation then further extends to $E^{\prime}$ with fixed field $E_{\bullet}^{\prime} \subsetneq E^{\prime}$ (see [43, Lemmas 2.1]). If $E / E_{\bullet}$ is unramified, then of course $E^{\prime} / E_{\bullet}^{\prime}$ is unramified and $f_{0}$ is odd. If $E / E_{\bullet}$ is ramified then, again as discussed in Section 3.1, either $f_{0}=1$ and so $E=E^{\prime}$, or else $f_{0}$ is even and $E^{\prime} / E_{\bullet}^{\prime}$ is unramified.

Proposition 3.13. A self-dual supercuspidal representation $\tilde{\pi}$ is of the same parity as its twist $\tilde{\pi}^{\prime}:=\tilde{\pi}|\operatorname{det}|^{\pi i /} f_{0} \log q_{E}$ if and only if $\tilde{\pi}$ is strongly ramified, i.e., $f_{0}=1$ and $E / E_{\bullet}$ is ramified.

Proof. Let $T / F$ be the maximal tamely ramified sub-extension of $E / F$, and $T^{\prime}$ be the unramified extension of $T$ of degree $f_{0}$ with fixed field $T_{\bullet}^{\prime}=T^{\prime} \cap E_{\bullet}^{\prime}$. Since $E^{\prime} / T^{\prime}$ is totally ramified of degree a power of $p$ which is odd, we have $T^{\prime} / T_{\bullet}^{\prime}$ is ramified if and only if $E^{\prime} / E_{\bullet}^{\prime}$ is ramified. The discussion prior to the proposition then implies that

$$
T^{\prime} / T_{\bullet}^{\prime} \text { is ramified if and only if } \tilde{\pi} \text { is strongly ramified. }
$$

From [18, Chapter 1], suppose the Langlands parameter of $\tilde{\pi}$ takes the form

$$
\operatorname{Ind}_{T / F}\left(\tilde{\alpha} \otimes \operatorname{Ind}_{T^{\prime} / T} \tilde{\xi}\right) \cong \operatorname{Ind}_{T^{\prime} / F}\left(\operatorname{Res}_{T^{\prime} / T} \tilde{\alpha} \otimes \tilde{\xi}\right),
$$

where $\tilde{\alpha}$ is an irreducible representation of $\mathcal{W}_{T}$, and $\tilde{\xi}$, as a character of $T^{\prime \times}$, is viewed as a character of $\mathcal{W}_{T^{\prime}}$. Denote by $|\cdot| \mathfrak{W}_{T^{\prime}}$ the unramified character of $\mathcal{W}_{T^{\prime}}$. The Langlands parameter of $\tilde{\pi}^{\prime}$ is therefore

$$
\operatorname{Ind}_{T^{\prime} / F}\left(\operatorname{Res}_{T^{\prime} / T} \tilde{\alpha} \otimes \tilde{\xi}\right) \otimes|\cdot|_{W_{F}}^{\pi i / \log q_{T^{\prime}}} \cong \operatorname{Ind}_{T^{\prime} / F}\left(\operatorname{Res}_{T^{\prime} / T} \tilde{\alpha} \otimes \tilde{\xi}|\cdot|{ }_{W_{T^{\prime}}}^{\pi i / \log q_{T^{\prime}}}\right) .
$$

Since the parity of a representation is preserved under induction, the proof is done by the remark before the proposition, that $|\cdot|_{T^{\prime}}^{\pi i / \log q_{T^{\prime}}}$ is conjugate-orthogonal if and only if $T^{\prime} / T_{\bullet}^{\prime}$ is ramified. 


\section{The coefficients}

The whole section is devoted to proving Theorem 3.4. It suffices to compute the values of $b_{w}$ for $w \in\{y, z\}$. We do not require that $\operatorname{char}(F)=0$.

We begin by recalling the explicit form of $J_{P}^{1}$. First of all, we recall and expand the subrings $\tilde{\mathfrak{H}}$ and $\tilde{\tilde{J}}$ in Section 2.2,

$$
\begin{aligned}
\tilde{\mathfrak{H}} & =\tilde{\mathfrak{H}}_{\Lambda, \beta}=\tilde{\mathfrak{A}}_{\Lambda, E}+\tilde{\mathfrak{P}}_{\Lambda, E_{1}}^{\left(r_{0} / 2\right)_{+}}+\cdots+\tilde{\mathfrak{P}}_{\Lambda, E_{d}}^{\left(r_{d-1} / 2\right)_{+}}+\tilde{\mathfrak{P}}_{\Lambda}^{\left(r_{d} / 2\right)_{+}}, \\
\tilde{\mathfrak{S}} & =\tilde{\widetilde{J}}_{\Lambda, \beta}=\tilde{\mathfrak{A}}_{\Lambda, E}+\tilde{\mathfrak{P}}_{\Lambda, E_{1}}^{r_{0} / 2}+\cdots+\tilde{\mathfrak{P}}_{\Lambda, E_{d}}^{r_{d-1} / 2}+\tilde{\mathfrak{P}}_{\Lambda}^{r_{d} / 2} .
\end{aligned}
$$

By [10, Proposition 1],

$$
\tilde{\mathcal{J}}_{\mathfrak{m}}^{1}=\left[\begin{array}{ccc}
\tilde{\widetilde{J}}^{1} & \tilde{\widetilde{J}}^{0} & \varpi_{E}^{-1} \tilde{\mathfrak{S}}^{1} \\
\tilde{\mathfrak{H}}^{1} & \tilde{\widetilde{J}}^{1} & \tilde{\widetilde{J}}^{0} \\
\varpi_{E} \tilde{\widetilde{J}}^{0} & \tilde{\mathfrak{S}}^{1} & \tilde{\tilde{\mathcal{J}}}^{1}
\end{array}\right] \cap A_{W} .
$$

In the strongly ramified case, since all exponents $r_{i}=2 s_{i}+1$, for $i=0, \ldots, d$, are odd, we have $\tilde{\widetilde{\Im}}_{\Lambda}=\tilde{\mathfrak{H}}_{\Lambda}$. We can show that $\tilde{\mathcal{F}}_{\mathfrak{m}}^{1}=\mathfrak{H}_{\mathfrak{m}}^{1}$ and $J_{\mathfrak{m}}^{1}=H_{\mathfrak{m}}^{1}=J_{P}^{1}$.

Note that we have chosen certain convenient normalizations of $T_{w}$, for $w \in\{y, z\}$, and $Z$ in the relation $t_{P}(Z)=T_{y} * T_{z}$ to simplify our calculations. Following [9], we may choose $T_{w}$ such that

$$
T_{w}\left(s_{w}\right) T_{w}\left(s_{w}^{-1}\right)=1,
$$

which can easily be shown to be equivalent to requiring that $T_{w}\left(s_{w}\right)^{2}=\lambda_{P}\left(s_{w}^{2}\right)$.

4.1. Computation of $\boldsymbol{b}_{\boldsymbol{y}}$. We use formula (2.10b) to compute $b_{y}$. Recall that it is

$$
b_{y}=\left(\operatorname{dim} \lambda_{P}\right)^{-1} \sum_{u \in \frac{s y J_{P}^{+} s y \cap J_{P} s_{y} J_{P}}{J_{P}}} \operatorname{tr} T_{y}(u) .
$$

The condition $J_{\mathfrak{m}}^{1}=H_{\mathfrak{m}}^{1}$ implies that $\operatorname{dim} \lambda_{P}=1$, so that the sum becomes

$$
b_{y}=\sum_{u \in\left(s_{y} J_{P}^{+}\right.} T_{\left.s_{y} \cap J_{P} s_{y} J_{P}\right) / J_{P}^{-}} T_{y}(u) .
$$

With the matrix presentation, we put $u=(X, Y)^{-}$with notation in Section 2.3, we hence compute $T_{y}\left((X, Y)^{-}\right)$for each $(X, Y)^{-} \in\left(s_{y} J_{P}^{+} s_{y} \cap J_{P} s_{y} J_{P}\right) / J_{P}^{-}$. We also recall (2.3) which we use repeatedly

$$
X^{\alpha} X=Y-{ }^{\alpha} Y .
$$

We take

$$
s_{y}=\left[\begin{array}{lll} 
& & I \\
& I & \\
I & &
\end{array}\right] \text {. }
$$

If

$$
(X, Y)^{-}=\left[\begin{array}{ccc}
I & & \\
{ }^{\alpha} X & I & \\
Y & X & I
\end{array}\right] \in s_{y} J_{P}^{+} s_{y} / J_{P}^{-},
$$


then from (4.1) we have

$$
X \in \tilde{\widetilde{J}}^{0} / \tilde{\mathfrak{H}}^{1} \quad \text { and } \quad Y \in \varpi_{E}^{-1} \tilde{\mathfrak{H}}^{1} / \varpi_{E} \tilde{\widetilde{J}}^{0} .
$$

Also, we write

$$
\operatorname{supp}\left(T_{y}\right)=J_{P} s_{y} J_{P}=J_{P}^{+} s_{y} J_{M} J_{P}^{+},
$$

so that $(X, Y)^{-} \in s_{y} J_{P}^{+} s_{y} \cap J_{P} s_{y} J_{P}$ can be written as

$$
\begin{aligned}
& {\left[\begin{array}{ccc}
I & & \\
{ }^{\alpha} X & I & \\
Y & X & I
\end{array}\right]=\left[\begin{array}{ccc}
I & { }^{\sigma} Y X & Y^{-1} \\
& I & \alpha\left({ }^{\sigma} Y X\right) \\
& & I
\end{array}\right]\left[\begin{array}{ccc} 
& & I \\
& I & \\
I & &
\end{array}\right]} \\
& \cdot\left[\begin{array}{ccc}
Y & & \\
& I-{ }^{\alpha} X Y^{-1} X & \\
& & { }^{\sigma} Y
\end{array}\right]\left[\begin{array}{ccc}
1 & Y^{-1} X & Y^{-1} \\
& 1 & { }^{\alpha}\left(Y^{-1} X\right) \\
& & 1
\end{array}\right] \text {, }
\end{aligned}
$$

or in simplified symbols,

$$
(X, Y)^{-}=\left({ }^{\sigma} Y X, Y^{-1}\right)^{+} \cdot s_{y} \cdot i_{M}\left(Y, I-{ }^{\alpha} X Y^{-1} X\right) \cdot\left(Y^{-1} X, Y^{-1}\right)^{+} .
$$

Lemma 4.1. Each coset in $\left(s_{y} J_{P}^{+} s_{y} \cap J_{P} s_{y} J_{P}\right) / J_{P}^{-}$has a representative of the form $\left(X, Y_{0}\left(I+Y^{\prime}\right)\right)^{-}$with $Y_{0} \in \mu_{E}$ and $X \in\left(\mathfrak{o}_{E} \backslash \mathfrak{p}_{E}\right) \bmod \mathfrak{p}_{E}$ such that $2 Y_{0} \equiv-X^{2} \bmod \mathfrak{p}_{E}$, and $I+Y^{\prime} \in \tilde{J}^{1}$.

Proof. The coset space $\tilde{\tilde{\mathcal{J}}}^{0} / \tilde{\mathfrak{H}}^{1}$ containing $X$, when viewed as a $\mathbf{k}_{F}$-vector space, takes the form $\left(\mathfrak{o}_{E} / \mathfrak{p}_{E}\right) \oplus\left(\tilde{\widetilde{J}}^{1} / \tilde{\mathfrak{H}}^{1}\right)$. If $r_{j}=2 s_{j}+1$ are odd, then $\tilde{\widetilde{J}}^{1}=\tilde{\mathfrak{H}}^{1}$, and so we can choose $X \in \mathfrak{o}_{E} \bmod \mathfrak{p}_{E}$. If furthermore $(X, Y)^{-} \in J_{P} s_{y} J_{P}$, then $Y$ is forced to belong to $\tilde{J}$ by (4.3), which allows us to choose $Y_{0} \in \mu_{E}$. The relation (4.2) implies that $2 Y_{0} \equiv-X^{2} \bmod \mathfrak{p}_{E}$, so that $X \notin \mathfrak{p}_{E}$, and $Y_{0}$ is uniquely determined by $X$. Hence the lemma follows.

From (4.3) we obtain

$$
T_{y}\left((X, Y)^{-}\right)=T_{y}\left(s_{y}\right) \tilde{\lambda}(Y) \lambda\left(I-{ }^{\alpha} X Y^{-1} X\right) .
$$

The following lemma shows that it is indeed a constant.

Lemma 4.2. For all $(X, Y)^{-} \in\left(s_{y} J_{P}^{+} s_{y} \cap J_{P} s_{y} J_{P}\right) / J_{P}^{-}$,

$$
T_{y}\left((X, Y)^{-}\right)=\tilde{\rho}(-2) \rho(-1) T_{y}\left(s_{y}\right) .
$$

Proof. Since now $X \in \tilde{J}$ is invertible, we have

$$
I-{ }^{\alpha} X Y^{-1} X=-{ }^{\alpha} X Y^{-1 \sigma} Y^{-1 \alpha} X^{-1}
$$

and so

$$
\lambda\left(I-{ }^{\alpha} X Y^{-1} X\right)=\rho(-1) \theta\left(Y^{\sigma} Y\right)^{-1}=\rho(-1) \theta\left(\left(I+Y^{\prime}\right)^{\sigma}\left(I+Y^{\prime}\right)\right)^{-1} .
$$

On the other hand, that $2 Y_{0} \equiv-X^{2} \bmod \mathfrak{p}_{E}$ implies that

$$
\tilde{\lambda}(Y)=\tilde{\rho}\left(Y_{0}\right) \tilde{\theta}\left(I+Y^{\prime}\right)=\tilde{\rho}(-2) \tilde{\theta}\left(I+Y^{\prime}\right) .
$$


Since $\tilde{\theta}$ and $\theta$ are related by (2.2), we have

$$
\tilde{\theta}\left(I+Y^{\prime}\right)=\theta\left(\left(I+Y^{\prime}\right)^{\sigma}\left(I+Y^{\prime}\right)\right),
$$

and the lemma follows.

By combining the above two lemmas with (3.3), we obtain

$$
b_{y}=\tilde{\rho}(-2) \rho(-1) T_{y}\left(s_{y}\right)\left(q_{E}-1\right)\left(c_{y} / q_{E}\right)^{1 / 2} .
$$

This proves Theorem 3.4(i).

4.2. Computation of $\boldsymbol{b}_{z}$. We use formula $(2.11 \mathrm{~b})$ to compute $b_{z}$. Recall that it is

$$
b_{z}=\left(\operatorname{dim} \lambda_{P}\right)^{-1} \sum_{u \in \frac{s_{z} J_{P}^{-} s_{z}^{-1} \cap J_{P} s_{z} J_{P}}{J_{P}^{+}}} \operatorname{tr} T_{z}(u)=\sum_{u \in \frac{s_{z} J_{P}^{-} s_{z}^{-1} \cap J_{P} s_{z} J_{P}}{J_{P}^{+}}} T_{z}(u)
$$

as $\operatorname{dim} \lambda_{P}=1$. We put $u=(X, Y)^{+}$with notation in Section 2.3, and compute $T_{z}\left((X, Y)^{+}\right)$ for each $(X, Y)^{+} \in\left(s_{z}^{-1} J_{P}^{-} s_{z} \cap J_{P} s_{z} J_{P}\right) / J_{P}^{+}$. We take

$$
s_{z}=\left[\begin{array}{ccc} 
& & -\varpi_{E}^{-1} I \\
\varpi_{E} I & &
\end{array}\right] .
$$

If

$$
(X, Y)^{+}=\left[\begin{array}{ccc}
I & X & Y \\
& I & { }^{\alpha} X \\
& & I
\end{array}\right] \in s_{z}^{-1} J_{P}^{-} s_{z} / J_{P}^{+}
$$

then we have

$$
X \in \varpi_{E}^{-1} \tilde{\mathfrak{H}}^{1} / \tilde{\tilde{J}}^{0} \quad \text { and } \quad Y \in \varpi_{E}^{-1} \tilde{\tilde{\mathcal{J}}}^{0} / \varpi_{E}^{-1} \tilde{\mathfrak{H}}^{1} .
$$

We also write

$$
\operatorname{supp}\left(T_{z}\right)=J_{P} s_{z} J_{P}=J_{P}^{-} J_{M} s_{z} J_{P}^{-},
$$

so that $(X, Y)^{+} \in J_{P} s_{Z} J_{P} \cap s_{z} J_{P}^{-} s_{z}$ can be written as

$$
\begin{aligned}
& {\left[\begin{array}{ccc}
I & X & Y \\
& I & { }^{\alpha} X \\
& & I
\end{array}\right]=\left[\begin{array}{ccc}
I & & \\
{ }^{\alpha} X Y^{-1} & I & \\
Y^{-1} & { }^{\alpha}\left({ }^{\alpha} X Y^{-1}\right) & I
\end{array}\right]\left[\begin{array}{ccc}
-Y \varpi_{E} & & \\
& I-{ }^{\alpha} X Y^{-1} X & \\
& & \\
& &
\end{array}\right.} \\
& {\left[\begin{array}{ccc} 
& & -\varpi_{E}^{-1} I \\
\varpi_{E} I & &
\end{array}\right]\left[\begin{array}{ccc}
I & & \\
{ }^{\alpha} X^{\sigma} Y & I & \\
Y^{-1} & \alpha\left({ }^{\alpha} X^{\sigma} Y\right) & I
\end{array}\right],}
\end{aligned}
$$

or in simplified symbols,

$$
(X, Y)^{+}=\left({ }^{\sigma} Y X, Y^{-1}\right)^{-} \cdot i_{M}\left(-Y \varpi_{E}, I-{ }^{\alpha} X Y^{-1} X\right) \cdot s_{z} \cdot\left(Y^{-1} X, Y^{-1}\right)^{-} .
$$

If we write $Y=y \varpi_{E}^{-1}\left(I+Y^{\prime}\right)$ with $y \in \mathfrak{o}_{E} \bmod \mathfrak{p}_{E}$ and $I+Y^{\prime} \in \tilde{J}^{1}=\tilde{H}^{1}$, then from (4.4) we can assume that $y \in \mu_{E}$. We hence obtain

$$
T_{z}\left((X, Y)^{+}\right)=\tilde{\rho}(-y) \tilde{\theta}\left(I+Y^{\prime}\right) \theta\left(I-{ }^{\alpha} X Y^{-1} X\right) T_{z}\left(s_{z}\right) .
$$

In the following subsections, we will expand and simplify $\tilde{\theta}\left(I+Y^{\prime}\right)$ and $\theta\left(I-{ }^{\alpha} X Y^{-1} X\right)$. 
4.2.1. Expanding simple characters. We fix an additive character $\psi$ of $F$ of conductor 1 , which means that it is trivial on $\mathfrak{p}_{F}$ but not ${ }^{\mathfrak{o}} F$. If $c \in A$ with $v_{\Lambda}(c)=-r$, then

$$
\psi_{c}: I+X \mapsto \psi_{F} \circ \operatorname{tr}_{A / F}(c X)
$$

defines a character on the compact subgroup $\tilde{U}_{\Lambda}^{(r / 2)}{ }_{+}$which looks additive, in the sense that

$$
\psi_{c}((I+X)(I+Y))=\psi_{c}(I+X+Y) .
$$

Recall from Section 3.1 that, given the skew simple stratum $\mathbf{s}=[\Lambda, r, 0, \beta]$, we have an approximation by skew simple strata $\left[\Lambda, r, r_{j}, \gamma_{j+1}\right], j=0, \ldots, d$, equivalent to $\left[\Lambda, r, r_{j}, \gamma_{j}\right]$. We denote $c_{j}=\gamma_{j}-\gamma_{j+1}$ (where we take $\gamma_{d+1}=0$ ) and $E_{j}=F\left[\gamma_{j}\right]$. We have $v_{\Lambda}\left(c_{j}\right)=-r_{j}$. Since $r_{j}=2 s_{j}+1$ is odd, $\left(r_{j} / 2\right)_{+}=s_{j}+1$.

The compact subgroup $\tilde{H}^{1}$ factorizes as (by [19, (3.1.15)] inductively)

$$
\tilde{U}_{E}^{1} \tilde{U}_{\Lambda, E_{1}}^{s_{0}+1} \cdots \tilde{U}_{\Lambda, E_{d}}^{s_{d-1}+1} \tilde{U}_{\Lambda}^{s_{d}+1} .
$$

Denote $\tilde{H}^{t}=\tilde{H}^{1} \cap \tilde{U}_{\Lambda}^{t}$ for all $t \geq 1$. A simple character $\tilde{\theta}=\tilde{\theta}_{0} \in \tilde{\mathscr{C}}(\Lambda, 0, \beta)$ takes the following inductive form: for $j=0, \ldots, d+1$,

$$
\left.\tilde{\theta}_{j}\right|_{\tilde{U}_{\Lambda, E_{j}}^{s_{j-1}+1}}=\tilde{\xi}_{j} \circ \operatorname{det}_{\tilde{B}_{j}}
$$

for some character $\tilde{\xi}_{j}$ of $U_{E_{j}}^{s_{j-1}+1}$, and for $j=0, \ldots, d$,

$$
\left.\tilde{\theta}_{j}\right|_{\tilde{H}^{s_{j}+1}}=\tilde{\theta}_{j+1} \psi_{c_{j}}
$$

for some simple character $\tilde{\theta}_{j+1} \in \tilde{e}\left(\Lambda, s_{j}, \gamma_{j}\right)$.

Similarly, $\theta$ is defined on

$$
H^{1}=U_{E / E_{\bullet}}^{1} U_{\Lambda, E_{1} / E_{\bullet}}^{s_{0}+1} \cdots U_{\Lambda, E_{d} / E_{\bullet} d}^{s_{d-1}+1} U_{\Lambda, F / F_{\bullet}}^{s_{d}+1}
$$

and takes the form

$$
\left.\theta_{j}\right|_{U_{\Lambda, E_{j} / E \bullet j}^{s_{j-1}+1}}=\xi_{j} \circ \operatorname{det}_{\tilde{B}_{j}}
$$

for some character $\xi_{j}$ of $U_{E_{j} / E_{\bullet j}}^{s_{j-1}+1}$ and $j=0, \ldots, d+1$, and

$$
\left.\theta_{j}\right|_{H^{s_{j}+1}}=\theta_{j+1} \psi_{c_{j} / 2}
$$

for some simple character $\theta_{j+1} \in \mathcal{C}\left(\Lambda, s_{j}, \gamma_{j}\right)$ and $j=0, \ldots, d$.

Since $\tilde{\theta}$ and $\theta$ are related by (2.2), we can and do assume similar relations between $\tilde{\theta}_{j}$ and $\theta_{j}$, and also between $\tilde{\xi}_{j}$ and $\xi_{j}$, i.e.,

$$
\xi_{j}=\left(\left.\tilde{\xi}_{j}\right|_{U_{E_{j} / E_{j}}^{s_{j-1}+1}}\right)^{1 / 2} .
$$

In the following subsections, we call the factors of $\tilde{\theta}$ involving $\tilde{\xi}_{j}$ the multiplicative parts of $\tilde{\theta}$, and those involving $\psi_{c_{j}}$ the additive parts, and similarly for $\theta$.

4.2.2. Cancellation of multiplicative parts. We recall that we have written

$$
Y=y \varpi_{E}^{-1}\left(I+Y^{\prime}\right),
$$

with $y \in \mu_{E}$ and $I+Y^{\prime} \in \tilde{H}^{1}$. We first compare the multiplicative parts of $\tilde{\theta}\left(I+Y^{\prime}\right)$ and 
$\theta\left(I-{ }^{\alpha} X Y^{-1} X\right)$ by rewriting

$$
\tilde{\theta}\left(I+Y^{\prime}\right)=\theta\left(\left(I+Y^{\prime}\right)^{\sigma}\left(I+Y^{\prime}\right)\right)=\rho(-1) \theta\left(I-X^{\alpha} X Y^{-1}\right)^{-1},
$$

and so we are actually comparing

$$
\theta\left(I-{ }^{\alpha} X Y^{-1} X\right) \text { and } \theta\left(I-X^{\alpha} X Y^{-1}\right) .
$$

Starting from the proposition below, we have to assume that

$$
E / F \text { is tamely ramified. }
$$

This condition allows us to assume that each field $E_{j}=F\left[\gamma_{j}\right]$, is contained in $E_{j-1}$ for all $j$, forming a tower of intermediate extensions between $E$ and $F$.

Lemma 4.3. The multiplicative parts of $\theta\left(I-{ }^{\alpha} X Y^{-1} X\right)$ and $\tilde{\theta}\left(I+Y^{\prime}\right)$ cancel with each other.

Proof. Let us temporarily write $W={ }^{\alpha} X Y^{-1}$. We have to compare $\theta(I-W X)$ and $\theta(I-X W)$. Let us first expand

$$
X=X_{0}+\cdots+X_{d+1},
$$

and similarly for $W$. For each $i=0, \ldots, d+1$, we write $X_{\leq i}=X_{0}+\cdots+X_{i}$, and similarly for $W_{\leq i}$. The part of the character $\theta(I-W X)$ involving $\xi_{i}$ is

$$
\xi_{i} \circ \operatorname{det}_{E_{i}}\left(\left(I-W_{\leq i-1} X_{\leq i-1}\right)^{-1}\left(I-W_{\leq i} X_{\leq i}\right)\right)
$$

and similarly for the part of $\theta\left(I-X^{\alpha} X Y^{-1}\right)$ involving $\xi_{i}$, with $W$ and $X$ exchanged. Due to the identity

$$
\operatorname{det}(I-W X)=\operatorname{det}(I-X W)
$$

these two parts are the same.

4.2.3. Choosing representatives. Here we will expand $X$ and $Y=y \varpi_{E}^{-1}\left(I+Y^{\prime}\right)$ such that the additive parts of $\tilde{\theta}\left(I+Y^{\prime}\right)$ and of $\theta\left(I-{ }^{\alpha} X Y^{-1} X\right)$ admit many simplifications.

The coset spaces $\varpi_{E}^{-1} \tilde{\mathfrak{H}}^{1} / \tilde{\mathcal{J}}^{0}$ and $\varpi_{E}^{-1} \tilde{\widetilde{\jmath}}^{0} / \varpi_{E}^{-1} \tilde{\mathfrak{H}}^{1}$ respectively containing $X$ and $Y$, when viewed as $\mathbf{k}_{F}$-vector spaces, take the form

$$
\varpi_{E}^{-1} \tilde{\mathfrak{H}}^{1} / \tilde{\widetilde{J}}^{0} \cong \bigoplus_{j=0}^{d} \mathfrak{W}_{z, j},
$$

where

$$
\mathfrak{W}_{z, j}=\tilde{\mathfrak{P}}_{\Lambda, E_{j+1}}^{-1+\left(r_{j} / 2\right)_{+}} /\left(\tilde{\mathfrak{P}}_{\Lambda, E_{j}}^{-1+\left(r_{j} / 2\right)_{+}}+\tilde{\mathfrak{P}}_{\Lambda, E_{j+1}}^{r_{j} / 2}\right),
$$

and

$$
\varpi_{E}^{-1} \tilde{\widetilde{J}}^{0} / \varpi_{E}^{-1} \tilde{\mathfrak{H}^{1}} \cong \mathbf{k}_{E}^{\prime} \oplus \bigoplus_{j=0}^{d} \mathfrak{W}_{z, j}^{\prime},
$$

where

$$
\mathbf{k}_{E}^{\prime} \cong \mathfrak{p}_{E}^{-1} / \mathfrak{o} E \quad \text { and } \quad \mathfrak{W}_{z, j}^{\prime}=\tilde{\mathfrak{P}}_{\Lambda, E_{j+1}}^{-1+\left(r_{j} / 2\right)} /\left(\tilde{\mathfrak{P}}_{\Lambda, E_{j}}^{-1+\left(r_{j} / 2\right)}+\tilde{\mathfrak{P}}_{\Lambda, E_{j+1}}^{-1+\left(r_{j} / 2\right)_{+}}\right) .
$$

Since each $r_{j}=2 s_{j}+1$ is odd, the summand $\mathfrak{W}_{z, j}^{\prime}$ is trivial. We expand $X=\sum_{j=0}^{d+1} X_{j}$ and 
$Y^{\prime}=\sum_{j=0}^{d+1} Y_{j}^{\prime}$ accordingly, first requiring that

$$
Y_{0}=y \varpi_{E}^{-1} \text { where } y \in \mu_{E}, \quad \text { and } \quad X_{j} \in \tilde{\mathfrak{P}}_{\Lambda, E_{j}}^{s_{j-1}} \bmod \tilde{\mathfrak{P}}_{\Lambda, E_{j}}^{s_{j-1}+1}+\tilde{\mathfrak{P}}_{\Lambda, E_{j-1}}^{s_{j-1}} \text { for } j>0 .
$$

With these fixed, we choose auxiliary data

$$
X_{0} \in \mathfrak{o}_{E} \quad \text { and } \quad Y_{j} \in \tilde{\mathfrak{P}}_{\Lambda, E_{j}}^{s_{j-1}} \text { for } j>0
$$

such that $X^{\alpha} X=Y-{ }^{\alpha} Y$ still holds. Eventually our main results are independent of these auxiliary choices, see (4.18) for example.

We require some notations. Let $E_{i}=F\left[\gamma_{i}\right]$ and $\tilde{B}_{i}$ the centralizer of $\gamma_{i}$ in $\tilde{A}$. For $i \geq 1$, we denote by

$$
\tilde{B}_{i-1}^{\perp} \text { the orthogonal complement of } \tilde{B}_{i-1} \text { in } \tilde{B}_{i}
$$

relative to the non-degenerate symmetric form

$$
(X, Y) \mapsto \operatorname{tr}_{\tilde{B}_{i} / E_{i}}(X Y) .
$$

We also write $1-\alpha: \tilde{A} \rightarrow \tilde{A}$ for the map $x \mapsto x-{ }^{\alpha} x$, whose image is denoted by $\tilde{A}^{-\alpha}$.

Lemma 4.4. Each coset in $\left(s_{z}^{-1} J_{P}^{-} s_{z} \cap J_{P} s_{z} J_{P}\right) / J_{P}^{+}$has a representative

$$
\left(X, y \varpi_{E}^{-1}\left(I+Y^{\prime}\right)\right)^{+}
$$

with expansions $X=\sum_{j=0}^{d+1} X_{j}$ and $Y^{\prime}=\sum_{j=0}^{d+1} Y_{j}^{\prime}$ such that

(i) $X_{0} \in \mathfrak{o}_{E}$ and $X_{j} \in \tilde{\mathfrak{P}}_{\Lambda, E_{j}}^{s_{j-1}}$ mod $\tilde{\mathfrak{P}}_{\Lambda, E_{j-1}}^{s_{j-1}}+\tilde{\mathfrak{P}}_{\Lambda, E_{j}}^{s_{j-1}+1}, j \geq 1$, that lies in $\tilde{\mathfrak{P}}_{\Lambda, E_{j}}^{s_{j-1}} \cap \tilde{B}_{j-1}^{\perp}$,

(ii) $Y_{0}^{\prime} \in \mathfrak{p}_{E}$ and $Y_{j}^{\prime} \in \tilde{\mathfrak{P}}_{\Lambda, E_{j}}^{s_{j-1}+1}$ for $j \geq 1$, with a decomposition

$$
Y_{j}^{\prime}=P_{j}+Q_{j}
$$

where $P_{j} \in \tilde{\mathfrak{P}}_{\Lambda, E_{j}}^{s_{j-1}+1} \cap \tilde{B}_{j-1}^{\perp}$ and $Q_{j} \in \tilde{\mathfrak{P}}_{\Lambda, E_{j}}^{r_{j-1}}$, satisfying the equations

$$
\sum_{\substack{\max (k, l)=j \\ k \neq l}} X_{k}^{\alpha} X_{l}=(1-\alpha)\left(y \varpi_{E}^{-1} P_{j}\right)
$$

and

$$
X_{j}^{\alpha} X_{j}=(1-\alpha)\left(y \varpi_{E}^{-1} Q_{j}\right)
$$

(Note that $P_{0}=0$.)

Proof. We can choose $X_{j}$, for $j \geq 1$, as stated using the commutative diagram

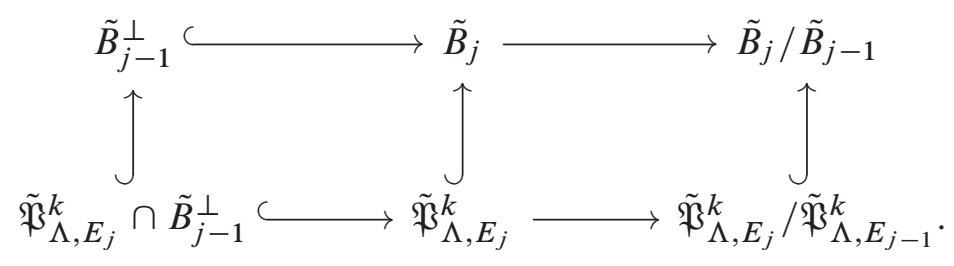

We see that the top row is an $\alpha$-equivariant isomorphism of $E_{j}$-spaces, which induces at the bottom row an $\alpha$-equivariant isomorphism $\tilde{\mathfrak{P}}_{\Lambda, E_{j}}^{k} \cap \tilde{B}_{j-1}^{\perp} \cong \tilde{\mathfrak{P}}_{\Lambda, E_{j}}^{k} / \tilde{\mathfrak{F}}_{\Lambda, E_{j-1}}^{k}$ of $\mathfrak{o}_{E_{j}}$-lattices 
for all $k \in \mathbb{Z}$. For choosing $P_{j}$, we notice that the left-hand side of (4.10) lies in $\tilde{B}_{j-1}^{\perp}$ by (4.12) as well as in the $(-\alpha)$-fixed point subspaces $\tilde{B}_{j}^{-\alpha}:=\tilde{B}_{j} \cap \tilde{A}^{-\alpha}$, and the restriction

$$
1-\alpha: \tilde{\mathfrak{P}}_{\Lambda, E_{j}}^{k} \cap \tilde{B}_{j-1}^{\perp} \rightarrow \tilde{\mathfrak{P}}_{\Lambda, E_{j}}^{k} \cap \tilde{B}_{j-1}^{\perp} \cap \tilde{B}_{j}^{-\alpha}
$$

is surjective. We can also choose $Q_{j}$ similarly. Now by putting $Y_{j}^{\prime}=P_{j}+Q_{j}$, we obtain

$$
\sum_{\max (k, l)=j} X_{k}^{\alpha} X_{l}=(1-\alpha)\left(y \varpi_{E}^{-1} Y_{j}^{\prime}\right),
$$

and summing it up for all $j$ yields $X^{\alpha} X=Y-{ }^{\alpha} Y$.

Here is a very simple consequence which will be frequently used later on: suppose that $T$ is either $X_{j}$ or $P_{j}$, for $j \geq 1$, as in Lemma 4.4, and $U$ is a product of elements in $B_{i}$ for $i<j$, then

$$
\operatorname{tr}_{\tilde{B}_{j} / E_{j}}(T U)=0 \text {. }
$$

4.2.4. Simplifying the additive parts. We will simplify the additive parts of $\tilde{\theta}\left(I+Y^{\prime}\right)$ and $\theta\left(I-{ }^{\alpha} X Y^{-1} X\right)$. Since each factor of the additive parts is a value of the character $\psi \circ \operatorname{tr}_{A / F}$, we will show that some of the inputs either lie in $\tilde{\mathfrak{P}}_{\Lambda}$ or have trace 0 by (4.12), so that their character values are 1.

Lemma 4.5. The additive part of $\tilde{\theta}\left(I+Y^{\prime}\right)$ is

$$
\prod_{j=0}^{d} \psi \circ \operatorname{tr}_{A / F}\left(\left(c_{j} y^{-1} \varpi_{E} / 2\right) X_{j+1}{ }^{\alpha} X_{j+1}\right) .
$$

Proof. In our calculation below, we have to switch between the additive expansion of $Y^{\prime} \in \tilde{\mathfrak{H}}_{\Lambda}^{1}$ given by $Y^{\prime}=\sum_{i=0}^{d+1} Y_{i}^{\prime}$ and the multiplicative expansion according to (4.6). Let us shorthand write

$$
\left(I+Y_{*}\right)_{0}^{k}=\left(I+Y_{0}\right) \cdots\left(I+Y_{k}\right) \text { and }\left(I+Y_{*}\right)_{k}^{0}=\left(I+Y_{k}\right) \cdots\left(I+Y_{0}\right)
$$

for $k=0, \ldots, d+1$, so that $Y=y \varpi_{E}^{-1}\left(I+Y_{*}\right)_{0}^{d+1}$. We have also written

$$
Y=y \varpi_{E}^{-1}\left(I+Y^{\prime}\right)
$$

where $Y^{\prime}=\sum_{i=0}^{d+1} Y_{i}^{\prime}$, such that

$$
Y_{i}^{\prime}=\left(I+Y_{*}\right)_{0}^{i-1} Y_{i}
$$

The additive part of $\tilde{\theta}\left(I+Y^{\prime}\right)$ is therefore equal to

$$
\prod_{\substack{i, j=0 \\ j<i}}^{d+1} \psi_{c_{j}}\left(I+Y_{i}\right)
$$

Note that each factor above can be rewritten as

$$
\psi_{c_{j}}\left(I+Y_{i}\right)=\psi \circ \operatorname{tr}_{A / F}\left(\frac{c_{j}}{2}\left(Y_{i}+{ }^{\alpha} Y_{i}\right)\right) .
$$


We write $\widehat{Y}_{i} \in \tilde{\mathfrak{P}}_{\Lambda, E_{i}}^{s_{i-1}+1}$ such that $\left(I+Y_{i}\right)\left(I+\widehat{Y}_{i}\right)=1$. We fix $j$ and rewrite the factors on the right-hand side of (4.15) as

$$
\psi \circ \operatorname{tr}_{A / F}\left(\frac{c_{j}}{2}\left(\left(I+\widehat{Y}_{*}\right)_{i-1}^{0} Y_{i}^{\prime}+{ }^{\alpha} Y_{i}^{\prime}\left(I-{ }^{\alpha} \widehat{Y}_{*}\right)_{0}^{i-1}\right)\right) .
$$

This expression admits a lot of simplification by writing $Y_{i}^{\prime}=P_{i}+Q_{i}$ as in Lemma 4.4. First of all, we have

$$
\psi \circ \operatorname{tr}_{A / F}\left(\frac{c_{j}}{2}\left(I+\widehat{Y}_{*}\right)_{i-1}^{0} P_{i}\right)=1
$$

since each summand has trace 0 by (4.12), and similarly

$$
\psi \circ \operatorname{tr}_{A / F}\left(\frac{c_{j}}{2}{ }^{\alpha} P_{i}\left(I-{ }^{\alpha} \widehat{Y}_{*}\right)_{0}^{i-1}\right)=1 .
$$

Moreover,

$$
\psi \circ \operatorname{tr}_{A / F}\left(\frac{c_{j}}{2}\left(I+\widehat{Y}_{*}\right)_{i-1}^{0} Q_{i}\right)=\psi \circ \operatorname{tr}_{A / F}\left(\frac{c_{j}}{2} Q_{i}\right),
$$

since $\frac{c_{j}}{2} Q_{i} \in \tilde{\mathfrak{V}}_{\Lambda}$ and all other summands (involving $\widehat{Y}_{*}$ ) lie in $\tilde{\mathfrak{P}}_{\Lambda}$. By the same reason, this term is non-trivial only when $i=j+1$. We have similarly

$$
\psi \circ \operatorname{tr}_{A / F}\left(\frac{c_{j} \alpha}{2} Q_{i}\left(I-{ }^{\alpha} \widehat{Y}_{*}\right)_{0}^{i-1}\right)= \begin{cases}\psi \circ \operatorname{tr}_{A / F}\left(\frac{c_{j}}{2} \alpha Q_{j+1}\right) & \text { if } i=j+1, \\ 1 & \text { otherwise. }\end{cases}
$$

Therefore, (4.14) is equal to

$$
\prod_{j=0}^{d} \psi \circ \operatorname{tr}_{A / F}\left(\frac{c_{j}}{2}\left(Q_{j+1}+{ }^{\alpha} Q_{j+1}\right)\right)
$$

and the lemma follows using (4.11).

Lemma 4.6. The additive part of $\theta\left(I-{ }^{\alpha} X Y^{-1} X\right)$ is

$$
\prod_{k=0}^{d} \psi \circ \operatorname{tr}_{A / F}\left(-\left(\frac{c_{k}}{2}\right)^{\alpha} X_{k+1} y^{-1} \varpi_{E} X_{k+1}\right) \text {. }
$$

Proof. If we expand

$$
I-{ }^{\alpha} X Y^{-1} X=\left(I+W_{*}\right)_{0}^{d+1}
$$

using (4.7) for some $W_{k} \in \tilde{\mathfrak{P}}_{\Lambda, E_{k}}^{s_{k-1}+1}$, then the additive part of $\theta\left(I-{ }^{\alpha} X Y^{-1} X\right)$ can be written as

$$
\prod_{\substack{k, l=0 \\ k<l}}^{d+1} \psi_{c_{k} / 2}\left(I+W_{l}\right)
$$

To express $I-{ }^{\alpha} X Y^{-1} X$ additively, we first denote, for every subset $S \subset\{0, \ldots, d+1\}$, a shorthand notation

$$
\left(\widehat{Y}_{*}\right)_{S}=\widehat{Y}_{i_{1}} \cdots \widehat{Y}_{i_{\# S}} \text { if } S=\left\{i_{1}, \ldots, i_{\# S}\right\} \text { listed in descending order. }
$$


We can expand $I-{ }^{\alpha} X Y^{-1} X$ as

$$
I-{ }^{\alpha} X Y^{-1} X=I-\sum_{i, j=0}^{d+1} \sum_{S}{ }^{\alpha} X_{i}\left(\widehat{Y}_{*}\right)_{S} y^{-1} \varpi_{E} X_{j},
$$

so that we can write $I-{ }^{\alpha} X Y^{-1} X=I+\sum_{k=0}^{d+1} W_{k}^{\prime}$ according to the additive expansion of $\mathfrak{S}_{\Lambda}^{1}$, where

$$
W_{k}^{\prime}=-\sum_{\max \{i, j, S\}=k}{ }^{\alpha} X_{i}\left(\widehat{Y}_{*}\right)_{S} y^{-1} \varpi_{E} X_{j} \in \tilde{\mathfrak{P}}_{\Lambda, E_{k}}^{s_{k-1}+1},
$$

We hence have

$$
W_{k}^{\prime}=\left(I+W_{*}\right)_{0}^{k-1} W_{k} .
$$

We now fix $k$ and simplify a sub-product from (4.16):

$$
\prod_{k<l} \psi_{c_{k} / 2}\left(I+W_{l}\right)
$$

by first expressing each of its factors as

$$
\psi_{c_{k} / 2}\left(I+W_{l}\right)=\psi \circ \operatorname{tr}_{A / F}\left(\frac{c_{k}}{2}\left(I+\widehat{W}_{*}\right)_{l-1}^{0} W_{l}^{\prime}\right)
$$

and using (4.17) to further expand the input for $\psi \circ \operatorname{tr}_{A / F}$ into summands of the form

$$
-\frac{c_{k}}{2}\left(I+\widehat{W}_{*}\right)_{l-1}^{0}{ }^{\alpha} X_{i}\left(\widehat{Y}_{*}\right)_{S} y^{-1} \varpi_{E} X_{j}
$$

The indices for this summand are $(i, j, S)$ such that $\max \{i, j, S\}=l>k$. In the following cases, this $(i, j, S)$-summand has zero trace.

(i) If $\max S<l$ and $i \neq j$, then since one of $i$ and $j$ is $l$, the summand has trace 0 by (4.12).

(ii) If max $S=l$ and exactly one of $i$ and $j$ is also $l$, then the summand lies in $\tilde{\mathfrak{P}}_{\Lambda}$.

(iii) If both $i, j<l$, then $\max S=l$. We write the summand as

$$
-\frac{c_{k}}{2}\left(I+\widehat{W}_{*}\right)_{l-1}^{0}{ }^{\alpha} X_{i} \widehat{Y}_{l}\left(\widehat{Y}_{*}\right)_{S-\{l\}} y^{-1} \varpi_{E} X_{j}
$$

and further expand $\widehat{Y}_{l}=\sum_{m=1}^{\infty}\left(-Y_{l}\right)^{m}$. Any summand involving $\left(-Y_{l}\right)^{m}$ with $m \geq 2$ lies in $\tilde{\mathfrak{P}}_{\Lambda}$, i.e., we remain to consider summands of the form

$$
\frac{c_{k}}{2}\left(I+\widehat{W}_{*}\right)_{l-1}^{0}{ }^{\alpha} X_{i} Y_{l}\left(\widehat{Y}_{*}\right)_{S-\{l\}} y^{-1} \varpi_{E} X_{j} .
$$

We then change $Y_{l}$ into $\left(I+\widehat{Y}_{*}\right)_{l-1}^{0} Y_{l}^{\prime}$ using (4.13) and decompose $Y_{l}^{\prime}=P_{l}+Q_{l}$. We then see that any summand involving $P_{l}$ has trace 0 , and any of those involving $Q_{l}$ lies in $\tilde{\mathfrak{P}}_{\Lambda}$.

(iv) The remaining case is $i=j=l$. The summand lies in $\tilde{\mathfrak{P}}_{\Lambda}$ except when $l=k+1$ and $S=\emptyset$, which is

$$
-\frac{c_{k}}{2}{ }^{\alpha} X_{k+1} y^{-1} \varpi_{E} X_{k+1}
$$


Therefore, we obtain

$$
\prod_{l>k} \psi_{c_{k} / 2}\left(I+W_{l}\right)=\psi \circ \operatorname{tr}_{A / F}\left(-\frac{c_{k}}{2}{ }^{\alpha} X_{k+1} y^{-1} \varpi_{E} X_{k+1}\right)
$$

and the lemma follows by multiplying the above equalities for all $k$ together.

By Lemmas 4.5 and 4.6, the additive part of $\tilde{\theta}\left(I+Y^{\prime}\right) \theta\left(I-{ }^{\alpha} X Y^{-1} X\right)$ is equal to

$$
\prod_{j=0}^{d} \psi \circ \operatorname{tr}_{A / F}\left(\left(y^{-1} \varpi_{E} / 2\right)\left(c_{j} X_{j+1}-X_{j+1} c_{j}\right)^{\alpha} X_{j+1}\right) .
$$

Note that it is independent of the auxiliary $X_{0}$, as expected from (4.9).

4.2.5. Non-degeneracy of a quadratic form. Define, for $j=0, \ldots, d$, a bilinear form on $\mathfrak{W}_{z, j}$ defined in (4.8) by

$$
\left.D_{j}(X, Y)=\operatorname{tr}_{A / F}\left(y^{-1} \varpi_{E}\left(X c_{j}-c_{j} X\right)^{\alpha} Y\right)\right) \bmod \mathfrak{p}_{F} \quad \text { for } X, Y \in \mathfrak{W}_{z, j}
$$

and put

$$
D=D_{0} \perp \cdots \perp D_{d},
$$

which defines a bilinear form on $\mathfrak{W}_{z}=\bigoplus_{j=0}^{d} \mathfrak{W}_{z, j}$ such that the decomposition (4.8) is orthogonal.

\section{Proposition 4.7. The quadratic form $D$ is non-degenerate.}

Proof. It suffices to show that each $D_{j}$ is non-degenerate. Hence we reduce to the situation where

- $E / F$ is a tamely ramified extension in $\tilde{A}$, generated by an element $c \in \tilde{A}$ with valuation $-r=-2 s-1$,

- $D(X, Y)=\operatorname{tr}_{A / F}\left(\varpi_{E} a_{c}(X) Y\right) \bmod \mathfrak{p}_{F}$, where $a_{c}: X \mapsto X c-c X$, is a bilinear form on $\mathfrak{W}:=\tilde{\mathfrak{P}}_{\Lambda}^{s} /\left(\tilde{\mathfrak{P}}_{\Lambda, E}^{s}+\tilde{\mathfrak{P}}_{\Lambda}^{s+1}\right)$.

We want to show that $D$ is non-degenerate, which is equivalent to showing that

$$
a_{c}(X) \in \tilde{\mathfrak{P}}_{\Lambda}^{-s} \Longrightarrow X \in \tilde{\mathfrak{P}}_{\Lambda, E}^{s}+\tilde{\mathfrak{P}}_{\Lambda}^{s+1} \text {. }
$$

This is implied by the definition of the critical exponent $[19,(1.4 .5)]$

$$
k_{0}(c, \Lambda)=\max \left\{k \in \mathbb{Z}: a_{c}^{-1} \tilde{\mathfrak{P}}_{\Lambda}^{k} \cap \tilde{\mathfrak{N}}_{\Lambda} \not \subset \tilde{\mathfrak{N}}_{\Lambda, E}+\tilde{\mathfrak{P}}_{\Lambda}\right\}
$$

and the minimality of $c$, i.e., $k_{0}(c, \Lambda)=v_{\Lambda}(c)=-r($ see $[19,(1.4 .15)])$.

4.2.6. A quadratic Gauss sum. By putting together (2.11), (4.5), Lemmas 4.3, 4.5, and 4.6, we obtain $b_{z}$, which is

$$
\begin{aligned}
& \tilde{\rho}(-1) T_{z}\left(s_{z}\right) \sum_{y \in \mu_{E}} \tilde{\rho}(y) \\
& \cdot \sum_{X \in \mathfrak{W}_{z}} \prod_{j=0}^{d} \psi \circ \operatorname{tr}_{A / F}\left(\left(y^{-1} \varpi_{E} / 2\right)\left(c_{j} X_{j+1}-X_{j+1} c_{j}\right)^{\alpha} X_{j+1}\right) .
\end{aligned}
$$


For a fixed $y \in \mu_{E}$, the above inner sum over $X \in \mathfrak{W}_{z}$ is a quadratic Gauss sum, defined on the $\mathbf{k}_{F}$-space $\mathfrak{W}_{z}$ equipped with the quadratic form in Proposition 4.7 which is non-degenerate. Note that, since $\Lambda$ is an $\mathfrak{D}_{E}$-lattice chain, $\mathfrak{W}_{z}$ is a $\mathbf{k}_{E}$-space as well. From basic properties of quadratic Gauss sums (cf. [15, Proposition 4.5]), the scalar $y^{-1} / 2$ can be factored out under the quadratic character as

$$
\left(\frac{y^{-1} / 2}{\mu_{E}}\right)^{\operatorname{dim}_{\mathbf{k}_{E}} \mathfrak{W}_{z}}=\left(\frac{2 y}{\mu_{E}}\right)^{\operatorname{dim}_{\mathbf{k}_{E}} \mathfrak{W}_{z}},
$$

so we can express (4.19) as

$$
\begin{aligned}
\tilde{\rho}(-1) & T_{z}\left(s_{z}\right) \sum_{y \in \mu_{E}} \tilde{\rho}(y)\left(\frac{2 y}{\mu_{E}}\right)^{\operatorname{dim}_{\mathbf{k}_{E}} \mathfrak{W}_{z}} \\
& \times \sum_{X \in \mathfrak{W}_{z}} \prod_{j=0}^{d} \psi \circ \operatorname{tr}_{A / F}\left(\varpi_{E}\left(c_{j} X_{j+1}-X_{j+1} c_{j}\right)^{\alpha} X_{j+1}\right) .
\end{aligned}
$$

Note that $\operatorname{dim}_{\mathbf{k}_{E}} \mathfrak{W}_{z}=f e-1$, where $e=e(E / F), f=f(E / F)$. In our situation that $e$ is odd, the sum over $y \in \mu_{E}$ is 0 when $\left.\tilde{\rho}\right|_{\mu_{E}}=\left(\frac{\cdot}{\mu_{E}}\right)^{f}$. Therefore

$$
b_{z}=0 \quad \text { when }\left.\tilde{\rho}\right|_{\boldsymbol{\mu}_{E}}=\left(\frac{\cdot}{\mu_{E}}\right)^{f} \text {. }
$$

We henceforth assume $\left.\tilde{\rho}\right|_{\boldsymbol{\mu}_{E}}=\left(\frac{\cdot}{\boldsymbol{\mu}_{E}}\right)^{f-1}$. The inner sum

$$
\sum_{X \in \mathfrak{W}_{z}} \prod_{j=0}^{d} \psi \circ \operatorname{tr}_{A / F}\left(\varpi_{E}\left(c_{j} X_{j+1}-X_{j+1} c_{j}\right)^{\alpha} X_{j+1}\right)
$$

equals a fourth root of unity, denoted by $\epsilon_{z}^{P}\left(\varpi_{E}, \mathbf{s}, \psi\right)$, times the positive number $\left(\# \mathfrak{W}_{z}\right)^{1 / 2}$. In our situation that $q$ is odd, since

$$
\epsilon_{z}^{P}\left(\varpi_{E}, \mathbf{s}, \psi\right)^{2}=\left(\frac{-1}{\mu_{E}}\right)^{\operatorname{dim}_{\mathbf{k}_{E}} \mathfrak{W}_{z}}=\left(\frac{-1}{\mu_{E}}\right)^{f-1}=(-1)^{\left(q^{f}-1\right)(f-1) / 2}=1,
$$

the normalized $\operatorname{sum} \epsilon_{z}^{P}\left(\varpi_{E}, \mathbf{s}, \psi\right)$ is actually a sign. Therefore,

$$
b_{z}=\tilde{\rho}(-2) \epsilon_{z}^{P}\left(\varpi_{E}, \mathbf{s}, \psi\right) T_{z}\left(s_{z}\right) \#\left(\mathfrak{W}_{z}\right)^{1 / 2}\left(q_{E}-1\right) \quad \text { when }\left.\tilde{\rho}\right|_{\mu_{E}}=\left(\frac{\cdot}{\mu_{E}}\right)^{f-1} .
$$

By noting that $\#\left(\mathfrak{W}_{z}\right)=c_{z} / q_{E}$, we finish the proof of Theorem 3.4 (ii).

Finally, we show that our main results are independent of the choices of the additive character $\psi$ and the uniformizer $\varpi_{E}$, as they should be.

Proposition 4.8. The following statements hold.

(i) The sign $\epsilon_{z}^{P}\left(\varpi_{E}, \mathbf{s}, \psi\right)$ is independent of the additive character $\psi$, hence we denote it by $\epsilon_{z}^{P}\left(\varpi_{E}, \mathbf{s}\right)$.

(ii) The relation $\tilde{\boldsymbol{\rho}}\left(\varpi_{E}\right)=\epsilon_{z}^{P}\left(\varpi_{E}, \mathbf{s}\right) \rho(-1)$ in Theorem 3.9 is independent of the choice of $\varpi_{E}$. 
Proof. For (i), we show that the $\operatorname{sign} \epsilon_{z}^{P}\left(\varpi_{E}, \mathbf{s}, \psi\right)$ is independent of the chosen additive character $\psi$ of $F$. Indeed if we replace $\psi$ by $\psi_{a}: F \rightarrow \mathbb{C}^{\times}, x \mapsto \psi(a x)$ for some $a \in F^{\times}$, then the Gauss sum is multiplied by $\left(\frac{a}{\mu_{E}}\right)^{f-1}$. Note that the character $\left(\frac{\dot{\mu_{E}}}{\boldsymbol{\mu}^{f-1}}\right.$ is non-trivial only when $f$ is even, so that $\left[\mu_{E}: \mu_{F}\right]=\left(q^{f}-1\right) /(q-1)$ is also even and $\left(\frac{\dot{\mu_{E}}}{\mu^{f-1}}\right.$ is always trivial on $\mu_{F}$.

To prove (ii), suppose that $\varpi_{E}$ is replaced by $u \varpi_{E}$ for some $u \in U_{E}$. It is easy to see that $\epsilon_{z}^{P}\left(u \varpi_{E}, \mathbf{s}\right)=\tilde{\rho}(u) \epsilon_{z}^{P}\left(\varpi_{E}, \mathbf{s}\right)$ from the expression (4.19).

Acknowledgement. This project began with a discussion between the second author and Shaun Stevens in the conference "Algebraisation and Geometrisation in the Langlands Programme" in Bristol, Spring 2016. The second author was offered a chance in the summer of 2016 to visit the first author and Colette Mœglin in Paris, supported by the funding of Michael Harris from the European Research Council and the hospitality of IHES. He was then supported by the postdoc fundings of Clifton Cunningham and Julia Gordon in the academic year 2016-2017, during the time a large part of the article was written. He would like to thank the above mentioned mathematicians, as well as Anne-Marie Aubert, Guy Henniart, Chung-Pang Mok, and Maarten Solleveld for discussions, and Ju-Lee Kim for providing him her preprint [30]. Both authors would like to thank Shaun Stevens for helpful mail discussions, and the referee for the careful reading.

\section{References}

[1] J. D. Adler and J. M. Lansky, Depth-zero base change for unramified U(2, 1), J. Number Theory 114 (2005), no. 2, 324-360.

[2] J.D. Adler and J.M. Lansky, Depth-zero base change for ramified U(2,1), Trans. Amer. Math. Soc. 362 (2010), no. 10, 5569-5599.

[3] J. Arthur, On local character relations, Selecta Math. (N.S.) 2 (1996), no. 4, 501-579.

[4] J. Arthur, The endoscopic classification of representations. Orthogonal and symplectic groups, American Mathematical Society Colloquium Publications 61, American Mathematical Society, Providence 2013.

[5] J. Arthur and L. Clozel, Simple algebras, base change, and the advanced theory of the trace formula, Ann. of Math. Stud. 120, Princeton University Press, Princeton 1989.

[6] M. Asgari, J. W. Cogdell and F. Shahidi, Local transfer and reducibility of induced representations of $p$-adic groups of classical type, in: Advances in the theory of automorphic forms and their $L$-functions, Contemp. Math. 664, American Mathematical Society, Providence (2016), 1-22.

[7] L. Blasco, Types, paquets et changement de base: l'exemple de $\mathrm{U}(2,1)\left(F_{0}\right)$. I. Types simples maximaux et paquets singletons, Canad. J. Math. 60 (2008), no. 4, 790-821.

[8] L. Blasco, Changements de base explicites des représentations supercuspidales de $\mathrm{U}(1,1)\left(F_{0}\right)$, Ann. Inst. Fourier (Grenoble) 60 (2010), no. 3, 905-938.

[9] L. Blasco and C. Blondel, Algèbres de Hecke et séries principales généralisées de $\mathrm{Sp}_{4}(F)$, Proc. Lond. Math. Soc. (3) 85 (2002), no. 3, 659-685.

[10] C. Blondel, Propagation de paires couvrantes dans les groupes symplectiques, Represent. Theory 10 (2006), 399-434.

[11] C. Blondel, Covers and propagation in symplectic groups, in: Functional analysis IX. Proceedings of the postgraduate school and conference (Dubrovnik 2005), University of Aarhus, Aarhus (2007), 16-31.

[12] C. Blondel, Représentation de Weil et $\beta$-extensions, Ann. Inst. Fourier (Grenoble) 62 (2012), no. 4, 1319-1366.

[13] C. Blondel, G. Henniart and S. Stevens, Jordan blocks of cuspidal representations of symplectic groups, Algebra Number Theory 12 (2018), no. 10, 2327-2386.

[14] C. J. Bushnell and G. Henniart, The essentially tame local Langlands correspondence. I, J. Amer. Math. Soc. 18 (2005), no. 3, 685-710. 
[15] C. J. Bushnell and G. Henniart, The essentially tame local Langlands correspondence. II. Totally ramified representations, Compos. Math. 141 (2005), no. 4, 979-1011.

[16] C. J. Bushnell and G. Henniart, The essentially tame local Langlands correspondence. III: The general case, Proc. Lond. Math. Soc. (3) 101 (2010), no. 2, 497-553.

[17] C. J. Bushnell and G. Henniart, The essentially tame Jacquet-Langlands correspondence for inner forms of GL(n), Pure Appl. Math. Q. 7 (2011), no. 3, 469-538.

[18] C. J. Bushnell and G. Henniart, To an effective local Langlands correspondence, Mem. Amer. Math. Soc. 231 (2014), no. 1087.

[19] C. J. Bushnell and P. C. Kutzko, The admissible dual of GL(N) via compact open subgroups, Ann. of Math. Stud. 129, Princeton University Press, Princeton 1993.

[20] C. J. Bushnell and P. C. Kutzko, Smooth representations of reductive $p$-adic groups: structure theory via types, Proc. Lond. Math. Soc. (3) 77 (1998), no. 3, 582-634.

[21] R. Ganapathy and S. Varma, On the local Langlands correspondence for split classical groups over local function fields, J. Inst. Math. Jussieu 16 (2017), no. 5, 987-1074.

[22] D. Goldberg, Some results on reducibility for unitary groups and local Asai $L$-functions, J. reine angew. Math. 448 (1994), 65-95.

[23] M. Harris and R. Taylor, The geometry and cohomology of some simple Shimura varieties. With an appendix by Vladimir G. Berkovich, Ann. of Math. Stud. 151, Princeton University Press, Princeton 2001.

[24] G. Henniart, Une preuve simple des conjectures de Langlands pour GL( $n)$ sur un corps $p$-adique, Invent. Math. 139 (2000), no. 2, 439-455.

[25] G. Henniart, Correspondance de Langlands et fonctions $L$ des carrés extérieur et symétrique, Int. Math. Res. Not. IMRN 2010 (2010), no. 4, 633-673.

[26] G. Henniart and R. Herb, Automorphic induction for GL( $n)$ (over local non-Archimedean fields), Duke Math. J. 78 (1995), no. 1, 131-192.

[27] R. E. Howe, Tamely ramified supercuspidal representations of $\mathrm{Gl}_{n}$, Pacific J. Math. 73 (1977), no. 2, 437-460.

[28] T. Kaletha, A. Minguez, S. W. Shin and P.-J. White, Endoscopic classification of representations: Inner forms of unitary groups, preprint 2014, https://arxiv.org/abs/1409.3731.

[29] J.-L. Kim, Hecke algebras of classical groups over p-adic fields. II, Compositio Math. 127 (2001), no. 2, 117-167.

[30] J.-L. Kim, Hecke algebras of classical groups over p-adic fields. III, prepint.

[31] R. E. Kottwitz and D. Shelstad, Foundations of twisted endoscopy, Astérisque (1999), no. 255.

[32] R. P. Langlands and D. Shelstad, On the definition of transfer factors, Math. Ann. 278 (1987), no. 1-4, 219-271.

[33] G. Laumon, M. Rapoport and U. Stuhler, $\mathscr{D}$-elliptic sheaves and the Langlands correspondence, Invent. Math. 113 (1993), no. 2, 217-338.

[34] J. Lust and S. Stevens, On depth zero L-packets for classical groups, Proc. Lond. Math. Soc. (3) 121 (2020), no. 5, 1083-1120.

[35] G. Lusztig, Irreducible representations of finite classical groups, Invent. Math. 43 (1977), no. 2, 125-175.

[36] M. Miyauchi and S. Stevens, Semisimple types for $p$-adic classical groups, Math. Ann. 358 (2014), no. 1-2, 257-288.

[37] C. Moglin, Sur la classification des séries discrètes des groupes classiques $p$-adiques: paramètres de Langlands et exhaustivité, J. Eur. Math. Soc. (JEMS) 4 (2002), no. 2, 143-200.

[38] C. Moeglin, Classification et changement de base pour les séries discrètes des groupes unitaires $p$-adiques, Pacific J. Math. 233 (2007), no. 1, 159-204.

[39] C. Moglin, Paquets stables des séries discrètes accessibles par endoscopie tordue; leur paramètre de Langlands, in: Automorphic forms and related geometry: assessing the legacy of I. I. Piatetski-Shapiro, Contemp. Math. 614, American Mathematical Society, Providence (2014), 295-336.

[40] C. Moeglin and D. Renard, Sur les paquets d'Arthur des groupes classiques et unitaires non quasi-déployés, in: Relative aspects in representation theory, Langlands functoriality and automorphic forms, Lecture Notes in Math. 2221, Springer, Cham (2018), 341-361.

[41] C. Moeglin and J.-L. Waldspurger, Stabilisation de la formule des traces tordue. Vol. 2, Progr. Math. 317, Birkhäuser, Cham 2016.

[42] C. P. Mok, Endoscopic classification of representations of quasi-split unitary groups, Mem. Amer. Math. Soc. 235 (2015), no. 1108.

[43] F. Murnaghan and J. Repka, Reducibility of some induced representations of $p$-adic unitary groups, Trans. Amer. Math. Soc. 351 (1999), no. 1, 193-210.

[44] J. D. Rogawski, Automorphic representations of unitary groups in three variables, Ann. of Math. Stud. 123, Princeton University Press, Princeton 1990. 
[45] P. Scholze, The local Langlands correspondence for $\mathrm{GL}_{n}$ over $p$-adic fields, Invent. Math. 192 (2013), no. 3, 663-715.

[46] F. Shahidi, A proof of Langlands' conjecture on Plancherel measures; complementary series for $p$-adic groups, Ann. of Math. (2) 132 (1990), no. 2, 273-330.

[47] A. J. Silberger, Special representations of reductive $p$-adic groups are not integrable, Ann. of Math. (2) 111 (1980), no. 3, 571-587.

[48] S. Stevens, Intertwining and supercuspidal types for $p$-adic classical groups, Proc. Lond. Math. Soc. (3) 83 (2001), no. 1, 120-140.

[49] S. Stevens, Semisimple characters for $p$-adic classical groups, Duke Math. J. 127 (2005), no. 1, 123-173.

[50] S. Stevens, The supercuspidal representations of $p$-adic classical groups, Invent. Math. 172 (2008), no. 2, 289-352.

[51] K.-F. Tam, Admissible embeddings of L-tori and the essentially tame local Langlands correspondence, Int. Math. Res. Not. IMRN 2016 (2016), no. 6, 1695-1775.

[52] K.-F. Tam, Some endoscopic properties of the essentially tame Jacquet-Langlands correspondence, Doc. Math. 21 (2016), 345-389.

[53] G. K.-F. Tam, Endoscopic classification of very cuspidal representations of quasi-split unramified unitary groups, Amer. J. Math. 140 (2018), no. 6, 1567-1638.

[54] J.-K. Yu, Construction of tame supercuspidal representations, J. Amer. Math. Soc. 14 (2001), no. 3, $579-622$.

Corinne Blondel, Université de Paris, Sorbonne Université, CNRS,

Institut de Mathématiques de Jussieu-Paris Rive Gauche, F-75013 Paris, France e-mail: corinne.blondel@imj-prg.fr

Geo Kam-Fai Tam, Department of Mathematics, IMAPP, Radboud University, Postbus 9010, 6500 GL Nijmegen, Netherlands e-mail: geotam@science.ru.nl

Eingegangen 16. März 2020, in revidierter Fassung 10. November 2020 